\title{
Hydrogen-Bonded Networks Based on Cobalt(II), Nickel(II), and Zinc(II) Complexes of N,N'-Diethylurea
}

\author{
Labrini Drakopoulou, ${ }^{1}$ Catherine P. Raptopoulou, ${ }^{2}$ \\ Aris Terzis, ${ }^{2}$ and Giannis S. Papaefstathiou ${ }^{3}$ \\ ${ }^{1}$ Department of Chemistry, University of Patras, 26504 Patras, Greece \\ ${ }^{2}$ Institute of Materials Science, National Centre of Scientific Research "Demokritos", 15310 Aghia Paraskevi Attikis, Greece \\ ${ }^{3}$ Laboratory of Inorganic Chemistry, Department of Chemistry, National and Kapodistrian University of Athens, Panepistimiopolis, \\ 15771 Zografou, Greece
}

Correspondence should be addressed to Giannis S. Papaefstathiou, gspapaef@chem.uoa.gr

Received 8 April 2010; Accepted 1 May 2010

Academic Editor: Spyros Perlepes

Copyright () 2010 Labrini Drakopoulou et al. This is an open access article distributed under the Creative Commons Attribution License, which permits unrestricted use, distribution, and reproduction in any medium, provided the original work is properly cited.

$\mathrm{N}, \mathrm{N}^{\prime}$-diethylurea (DEU) was employed as a ligand to form the octahedral complexes $\left[\mathrm{M}(\mathrm{DEU})_{6}\right]^{2+}(\mathrm{M}=\mathrm{Co}, \mathrm{Ni}$ and $\mathrm{Zn})$. Compounds $\left[\mathrm{Co}(\mathrm{DEU})_{6}\right]\left(\mathrm{BF}_{4}\right)_{2}(\mathbf{1}),\left[\mathrm{Co}(\mathrm{DEU})_{6}\right]\left(\mathrm{CIO}_{4}\right)_{2}(\mathbf{2}),\left[\mathrm{Ni}(\mathrm{DEU})_{6}\right]\left(\mathrm{CIO}_{4}\right)_{2}(\mathbf{3})$, and $\left[\mathrm{Zn}(\mathrm{DMU})_{6}\right](\mathrm{CIO})_{4}(4){ }^{2}$ have been prepared from the reactions of DEU and the appropriate hydrated metal(II) salts in EtOH in the presence of 2,2dimethoxypropane. Crystal structure determinations demonstrate the existence of $\left[\mathrm{M}(\mathrm{DEU})_{6}\right]^{2+}$ cations and $\mathrm{CIO}_{4}{ }^{-}$(in 2-4) or $\mathrm{BF}_{4}{ }^{-}$(in 1) counterions. The $\left[\mathrm{M}(\mathrm{DEU})_{6}\right]^{2+}$ cations in the solid state are stabilized by a pseudochelate effect due to the existence of six strong intracationic $\mathrm{N}-\mathrm{H} \cdots \mathrm{O}_{(\mathrm{DEU})}$ hydrogen bonds. The $\left[\mathrm{M}(\mathrm{DEU})_{6}\right]^{2+}$ cations and counterions self-assemble to form hydrogen-bonded 2D architectures in 2-4 that conform to the kgd (kagome dual) network, and a 3D hydrogen-bonded rtl (rutile) network in $\mathbf{1}$. The nature of the resulting supramolecular structures is influenced by the nature of the counter-ion. The complexes were also characterized by vibrational spectroscopy (IR).

\section{Introduction}

In 1828, Wöhler attempted to synthesize ammonium cyanate by reacting silver isocyanate (AgNCO) with ammonium chloride $\left(\mathrm{NH}_{4} \mathrm{Cl}\right)$. The outcome of this failed attempt was urea $\mathrm{H}_{2} \mathrm{NCONH}_{2}$ (U, Scheme 1) which represents the first organic molecule synthesized in the laboratory from purely inorganic materials [1]. Urea has also been recognized as the first organic molecule that was synthesized without the involvement of any living system [1]. Nowadays, urea represents not only an important molecule in biology [2] but also an important raw material in chemical industry [3].

Restricting further discussion to the coordination chemistry of urea and its substituted derivatives, metal-urea complexes have attracted considerable interest since the discovery of the active site of urease, a metalloenzyme that catalyzes the hydrolysis of urea into carbon dioxide and ammonia [4, 5]. Considerable efforts have been devoted to devise useful bioinorganic models for the active site of urease and provide information for the intermediates and its catalytic mechanism. That in turn drove to the structural and spectroscopic characterization of many metal-urea complexes [6]. Urea usually coordinates as a monodentate ligand through the oxygen atom, forming a $\mathrm{C}=\mathrm{O} \cdots \mathrm{M}$ angle considerably smaller than $180^{\circ}$, in accordance with the $s p^{2}$ hybridization of the $\mathrm{O}$ atom ( $\mathrm{A}$ in Scheme 2). The rare $\mathrm{N}, \mathrm{O}$-bidentate coordination mode (B in Scheme 2) has been found in a very limited number of cases $[7,8]$, while in $\left[\mathrm{Hg}_{2} \mathrm{Cl}_{4} \mathrm{U}_{2}\right]$ each $\mathrm{U}$ molecule bridges the two $\mathrm{Hg}^{\mathrm{II}}$ atoms through the oxygen atom [9] (C in Scheme 2). Of particular chemical/biological interest is the ability of $\mathrm{U}$ to undergo metal-promoted deprotonation $[4,10]$; the monoanionic ligand $\mathrm{H}_{2} \mathrm{NCONH}^{-}$adopts the $\mu_{2}$ (D in Scheme 2) and $\mu_{3}$ ( $\mathbf{E}$ in Scheme 2) coordination modes. 
<smiles>NC(N)=O</smiles>

$\mathrm{U}$<smiles>CNC(=O)NC</smiles>

DMU<smiles>CCNC(=O)NCC</smiles>

DEU

Scheme 1: Ligands discussed in the text $\left(\mathrm{U}=\right.$ urea, $\mathrm{DMU}=\mathrm{N}, \mathrm{N}^{\prime}$-dimethylurea and DEU $=\mathrm{N}^{\mathrm{N}} \mathrm{N}^{\prime}$-diethylurea).<smiles>[M]OC(N)=O</smiles>

(A)<smiles></smiles>

(D)<smiles></smiles>

(B)<smiles>[M]NC(=O)N[M]</smiles>

(E)<smiles>[M]OC(N)N</smiles>

(C)<smiles></smiles>

$(\mathbf{F})$

Scheme 2: The crystallographically established coordination modes of urea (U) and its symmetrically substituted alkyl derivatives (RHNCONHR).

The N,N'-alkyl symmetrically substituted derivatives of urea (RHNCONHR), such as the $\mathrm{N}^{\mathrm{N}} \mathrm{N}^{\prime}$-dimethylurea (DMU) and N,N'-diethylurea (DEU) (Scheme 1) have only been found to coordinate as monodentate ligands through the oxygen atom ( $\mathrm{F}$ in Scheme 2).

Urea and its substituted derivatives have been extensively studied within the frame of organic crystal engineering due to their ability to form extended hydrogen bonded frameworks. In particular, symmetrically substituted ureas (i.e., RHNCONHR) form $\alpha$-networks with each urea molecule donating two hydrogen bonds and "chelating" the carbonyl oxygen of the next molecule in the network. In contrast to the great number of studies concerning free ureas [11-15], little is known about the supramolecular structures based on hydrogen bonding interactions between simple metalureas complexes. Over the last decade, we have been studying the coordination chemistry of urea and its symmetrically substituted derivative DMU [16-21]. In all cases, ureas form stable complexes which are further connected to create extended frameworks by intermolecular/interionic hydrogen bond interactions. Despite the large number of metal-urea complexes which have been structurally characterized, the metal-DMU complexes are considerably less studied while there only three reports with crystal structures of metalDEU complexes [22-24]. In this report we present our first results from the study of metal-DEU complexes, extending the known crystal structures of metal-DEU complexes to seven.

\section{Experiments}

All manipulations were performed under aerobic conditions using materials and solvents as received. IR spectra were recorded on a Perkin-Elmer PC16 FT-IR spectrometer with samples prepared as $\mathrm{KBr}$ pellets. $\mathrm{C}, \mathrm{H}$ and $\mathrm{N}$ elemental analyses were performed with a Carlo Erba EA 108 analyzer.

Caution. Perchlorate salts are potentially explosive. Although no detonation tendencies have been observed in our experiments, caution is advised and handling of only small quantities is recommended.

[Co $\left.(D E U)_{6}\right]\left(B F_{4}\right)_{2}(\mathbf{1})$. A pink solution of $\mathrm{Co}\left(\mathrm{BF}_{4}\right)_{2} \cdot 6 \mathrm{H}_{2} \mathrm{O}$ $(0.68 \mathrm{~g}, 2.0 \mathrm{mmol})$ in $\mathrm{EtOH}(30 \mathrm{~mL})$ and dimethoxypropane (DMP) $(2.5 \mathrm{~mL})$ was refluxed for 20 minutes, cooled to room temperature and then treated with solid DEU ( $1.40 \mathrm{~g}$, $12 \mathrm{mmol}$ ). No noticeable colour change occurred. The reaction mixture was refluxed for a further 15 minutes, cooled to room temperature, and layered with $\mathrm{Et}_{2} \mathrm{O}(30 \mathrm{~mL})$. Slow mixing gave pink crystals suitable for X-ray crystallography, which were collected by filtration, washed with cold EtOH $(2 \mathrm{~mL})$ and $\mathrm{Et}_{2} \mathrm{O}$, and dried in vacuo over $\mathrm{CaCl}_{2}$. Typical yields were in the $70-80 \%$ range. Found \%: C, 38.96; H, 7.59; N, 17.90. Calc \% for $\mathrm{C}_{30} \mathrm{H}_{72} \mathrm{~N}_{12} \mathrm{O}_{6} \mathrm{CoB}_{2} \mathrm{~F}_{8}$ : C, 38.77; $\mathrm{H}, 7.81 ; \mathrm{N}, 18.08$. IR data $\left(\mathrm{KBr}, \mathrm{cm}^{-1}\right): 3332 \mathrm{sb}, 2976 \mathrm{~s}$, $2934 \mathrm{~m}, 2878 \mathrm{~m}, 1626$ vs, 1576 vs, $1482 \mathrm{w}, 1454 \mathrm{~m}, 1380 \mathrm{~m}$, 
$1338 \mathrm{~m}, 1294 \mathrm{~m}, 1160 \mathrm{~m}, 1142 \mathrm{~m}, 1110 \mathrm{sb}, 1032 \mathrm{~s}, 922 \mathrm{w}$, 890 w, $768 \mathrm{~m}, 578 \mathrm{mb}$.

$\left[\mathrm{Co}(\mathrm{DEU})_{6}\right]\left(\mathrm{ClO}_{4}\right)_{2}$ (2). A pink-red solution of $\mathrm{Co}\left(\mathrm{ClO}_{4}\right)_{2}$. $6 \mathrm{H}_{2} \mathrm{O}(0.73 \mathrm{~g}, 2.0 \mathrm{mmol})$ in $\mathrm{EtOH}(20 \mathrm{~mL})$ and dimethoxypropane (DMP) $(2.5 \mathrm{~mL})$ was refluxed for 20 minutes, cooled to room temperature and then treated with solid DEU $(1.40 \mathrm{~g}, 12 \mathrm{mmol})$. No noticeable colour change occurred. The reaction mixture was refluxed for a further $20 \mathrm{~min}$ utes, cooled to room temperature, and layered with $\mathrm{Et}_{2} \mathrm{O}$ $(50 \mathrm{~mL})$. Slow mixing gave pink crystals suitable for X-ray crystallography, which were collected by filtration, washed with cold $\mathrm{EtOH}(2 \mathrm{~mL})$ and $\mathrm{Et}_{2} \mathrm{O}$, and dried in vacuo over $\mathrm{CaCl}_{2}$. Typical yields were in the $75-85 \%$ range. Found \%: C, 37.92; $\mathrm{H}, 7.49 ; \mathrm{N}, 17.80$. Calc $\%$ for $\mathrm{C}_{30} \mathrm{H}_{72} \mathrm{~N}_{12} \mathrm{O}_{14} \mathrm{CoCl}_{2}$ : C, 37.74; $\mathrm{H}, 7.60 ; \mathrm{N}, 17.60$. IR data $\left(\mathrm{KBr}, \mathrm{cm}^{-1}\right): 3332 \mathrm{sb}, 2972 \mathrm{~s}$, $2934 \mathrm{~m}, 2876 \mathrm{w}, 1628 \mathrm{vs}, 1570 \mathrm{vs}, 1482 \mathrm{w}, 1452 \mathrm{w}, 1378 \mathrm{w}$, $1338 \mathrm{w}, 1296 \mathrm{w}, 1264 \mathrm{w}, 1142 \mathrm{~s}, 1114 \mathrm{~s}, 1086 \mathrm{~s}, 922 \mathrm{w}, 890 \mathrm{w}$, $768 \mathrm{w}, 626 \mathrm{~m}$.

$\left[\mathrm{Ni}(\mathrm{DEU})_{6}\right]\left(\mathrm{ClO}_{4}\right)_{2} \quad$ (3). A pale green solution of $\mathrm{Ni}\left(\mathrm{ClO}_{4}\right)_{2} \cdot 6 \mathrm{H}_{2} \mathrm{O}(0.73 \mathrm{~g}, 2.0 \mathrm{mmol})$ in $\mathrm{EtOH}(15 \mathrm{~mL})$ and dimethoxypropane (DMP) $(2.5 \mathrm{~mL})$ was refluxed for 15 minutes, cooled to room temperature and then treated with solid DEU ( $1.40 \mathrm{~g}, 12 \mathrm{mmol})$. No noticeable colour change occurred. The reaction mixture was refluxed for a further 20 minutes, cooled to room temperature, and layered with $\mathrm{Et}_{2} \mathrm{O}$ $(30 \mathrm{~mL})$. Slow mixing gave green crystals suitable for X-ray crystallography, which were collected by filtration, washed with cold $\mathrm{EtOH}(2 \mathrm{~mL})$ and $\mathrm{Et}_{2} \mathrm{O}$, and dried in vacuo over $\mathrm{CaCl}_{2}$. Typical yields were in the $75-85 \%$ range. Found \%: C, 37.90; $\mathrm{H}, 7.45 ; \mathrm{N}, 17.82$. Calc \% for $\mathrm{C}_{30} \mathrm{H}_{72} \mathrm{~N}_{12} \mathrm{O}_{14} \mathrm{NiCl}_{2}$ : C, 37.75; H, 7.60; N, 17.61. IR data $\left(\mathrm{KBr}, \mathrm{cm}^{-1}\right): 3328 \mathrm{sb}$, $2976 \mathrm{~m}, 2934 \mathrm{w}, 2876 \mathrm{w}, 1636 \mathrm{vs}, 1570 \mathrm{vs}, 1508 \mathrm{w}, 1450 \mathrm{~m}$, $1380 \mathrm{w}, 1334 \mathrm{w}, 1268 \mathrm{~m}, 1146 \mathrm{~s}, 1118 \mathrm{~s}, 1086 \mathrm{~s}, 922 \mathrm{w}, 772 \mathrm{w}$, $626 \mathrm{~m}$.

$\left[\mathrm{Zn}(\mathrm{DEU})_{6}\right]\left(\mathrm{ClO}_{4}\right)_{2}$ (4). A colourless solution of $\mathrm{Zn}($ $\left.\mathrm{ClO}_{4}\right)_{2} \cdot 6 \mathrm{H}_{2} \mathrm{O}(0.74 \mathrm{~g}, 2.0 \mathrm{mmol})$ in $\mathrm{EtOH}(10 \mathrm{~mL})$ and dimethoxy-propane (DMP) $(2.5 \mathrm{~mL})$ was refluxed for 20 minutes, cooled to room temperature, and then treated with solid DEU ( $1.40 \mathrm{~g}, 12 \mathrm{mmol})$. The colourless reaction mixture was refluxed for a further 20 minutes, cooled to room temperature, and layered with $\mathrm{Et}_{2} \mathrm{O}(25 \mathrm{~mL})$. Slow mixing gave colourless crystals suitable for X-ray crystallography, which were collected by filtration, washed with cold $\mathrm{EtOH}(2 \mathrm{~mL})$ and $\mathrm{Et}_{2} \mathrm{O}$, and dried in vacuo over $\mathrm{CaCl}_{2}$. Typical yields were in the $75-85 \%$ range. Found \%: $\mathrm{C}$, 37.62; $\mathrm{H}, 7.39 ; \mathrm{N}, 17.60$. Calc $\%$ for $\mathrm{C}_{30} \mathrm{H}_{72} \mathrm{~N}_{12} \mathrm{O}_{14} \mathrm{ZnCl}_{2}$ : C, $37.49 ; \mathrm{H}, 7.55 ; \mathrm{N}, 17.49$. IR data $\left(\mathrm{KBr}, \mathrm{cm}^{-1}\right): 3340 \mathrm{sb}, 2972 \mathrm{~s}$, $2932 \mathrm{~m}, 2876 \mathrm{w}, 1624 \mathrm{vs}, 1582 \mathrm{vs}, 1484 \mathrm{w}, 1456 \mathrm{w}, 1380 \mathrm{w}$, 1334 w, 1262 m, 1144 s, 1114 s, 1088 s, 924 w, 772 w, 636 m.

2.1. X-ray Crystallography. X-ray data were collected at $298 \mathrm{~K}$ using a Crystal Logic Dual Goniometer diffractometer with graphite-monochromated Mo- $K_{a}$ radiation $(\lambda=0.71073 \AA)$. Lorentz, polarization, and $\Psi$-scan absorption corrections were applied using Crystal Logic software. The structures

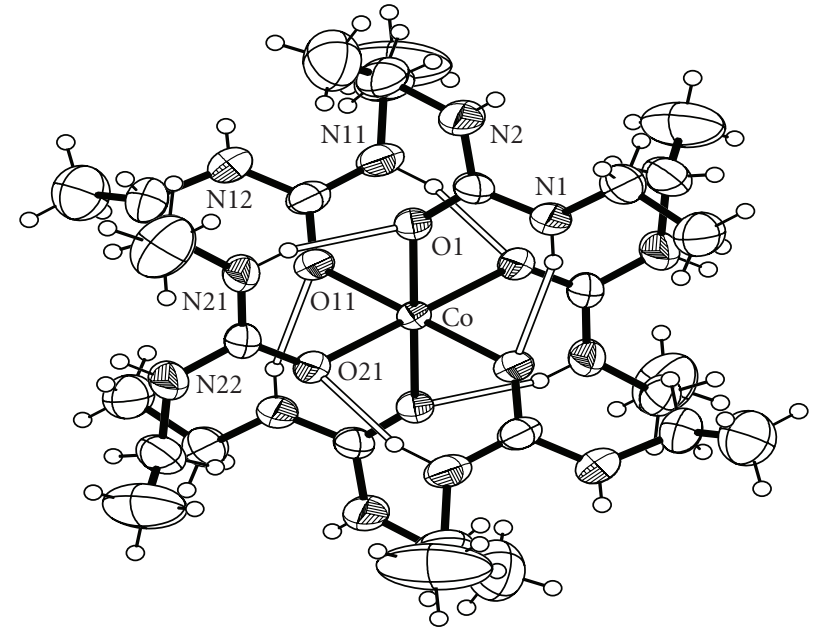

FIgURE 1: An ORTEP representation of the cation $\left[\mathrm{Co}(\mathrm{DEU})_{6}\right]^{2+}$ present in complex 1. Open bonds indicate intramolecular hydrogen bonds. The symmetry-equivalent atoms are not labeled.

were solved by direct methods using SHELXS-86 [25] and refined by full-matrix least-squares techniques on $F^{2}$ with SHELXL-97 [26]. Details of the data collection and refinement are given in Table 1. Topological analysis of the nets was performed using TOPOS program package [27, 28].

\section{Results and Discussion}

3.1. Synthetic Comments. The preparation of the three complexes reported here is summarized in (1):

$$
\mathrm{MX}_{2} \cdot 6 \mathrm{H}_{2} \mathrm{O}+6 \mathrm{DEU} \underset{\mathrm{T}, \mathrm{DMP}}{\stackrel{\text { EtOH }}{\longrightarrow}}\left[\mathrm{M}(\mathrm{DEU})_{6}\right] \mathrm{X}_{2}+6 \mathrm{H}_{2} \mathrm{O}
$$

$\mathrm{M}=\mathrm{Co}, \mathrm{X}=\mathrm{BF}_{4}(\mathbf{1}) ; \mathrm{M}=\mathrm{Co}, \mathrm{X}=\mathrm{ClO}_{4}(2) ; \mathrm{M}=\mathrm{Ni}, \mathrm{X}=$ $\mathrm{ClO}_{4}(3) ; \mathrm{M}=\mathrm{Zn}, \mathrm{X}=\mathrm{ClO}_{4}(4)$.

2,2-dimethoxypropane (DMP), is a known dehydrating agent which under heating eliminates the possibility of $\left[\mathrm{M}\left(\mathrm{H}_{2} \mathrm{O}\right)_{6}\right]^{2+}$ formation in solution.

Complexes 1-4 seem to be the only products from the $\mathrm{MX}_{2} \cdot 6 \mathrm{H}_{2} \mathrm{O} / \mathrm{DEU}$ reaction systems $\left(\mathrm{M}=\mathrm{Co}, \mathrm{Ni}, \mathrm{Zn}, \mathrm{X}=\mathrm{ClO}_{4}\right.$ and $\left.\mathrm{M}=\mathrm{Co}, \mathrm{X}=\mathrm{BF}_{4}\right)$. Changing the solvent from $\mathrm{EtOH}$ to $\mathrm{MeCN}$ to THF and $\mathrm{Me}_{2} \mathrm{CO}$ as well as the DEU : $\mathrm{M}^{\mathrm{II}}$ reaction ratio from $6: 1$ to $12: 1,8: 1,4: 1$ and $3: 1$ does not seem to influence the identity of the products.

3.2. Description of Structures. Bond distances and angles for complexes 1, 2, 3 and 4 are listed in Tables 2, 3, 4, and 5 , respectively. ORTEP plots of the cations $\left[\mathrm{Co}(\mathrm{DEU})_{6}\right]^{2+}$, $\left[\mathrm{Ni}(\mathrm{DEU})_{6}\right]^{2+}$, and $\left[\mathrm{Zn}(\mathrm{DEU})_{6}\right]^{2+}$ present in complexes $\mathbf{1}$, 2, 3, and 4 are shown in Figures 1, 2, 3, and 4, respectively. Details of the hydrogen bonds of $1,2,3$, and 4 are provided in Tables 6, 7, 8, and 9, respectively. Complexes 2, 3, and 4 crystallise in the triclinic space group $P \overline{1}$ and are isostructural. Complex $\mathbf{1}$ crystallizes in the monoclinic space group $P 2_{1} / \mathrm{c}$. The structures of $\mathbf{2 - 4}$ consist of almost perfect octahedral $\left[\mathrm{M}(\mathrm{DEU})_{6}\right]^{2+}$ cations and $\mathrm{ClO}_{4}{ }^{-}$counterions, while the same $\left[\mathrm{M}(\mathrm{DEU})_{6}\right]^{2+}$ cation and $\mathrm{BF}_{4}^{-}$anions are 
TABLE 1: Crystal data and structure refinement for $\mathbf{1 - 4}$.

\begin{tabular}{|c|c|c|c|c|}
\hline Compound & 1 & 2 & 3 & 4 \\
\hline Empirical formula & $\mathrm{C}_{30} \mathrm{H}_{72} \mathrm{~B}_{2} \mathrm{CoF}_{8} \mathrm{~N}_{12} \mathrm{O}_{6}$ & $\mathrm{C}_{30} \mathrm{H}_{72} \mathrm{CoCl}_{2} \mathrm{~N}_{12} \mathrm{O}_{14}$ & $\mathrm{C}_{30} \mathrm{H}_{72} \mathrm{NiCl}_{2} \mathrm{~N}_{12} \mathrm{O}_{14}$ & $\mathrm{C}_{30} \mathrm{H}_{72} \mathrm{ZnCl}_{2} \mathrm{~N}_{12} \mathrm{O}_{14}$ \\
\hline Formula weight & 929.52 & 954.81 & 954.59 & 961.26 \\
\hline Crystal size & $0.10 \times 0.20 \times 0.20$ & $0.10 \times 0.15 \times 0.20$ & $0.10 \times 0.15 \times 0.20$ & $0.10 \times 0.20 \times 0.20$ \\
\hline Crystal system & monoclinic & triclinic & triclinic & triclinic \\
\hline Space group & $P 2_{1} / \mathrm{c}$ & $P \overline{1}$ & $P \overline{1}$ & $P \overline{1}$ \\
\hline$\theta$ range for data & $1.85 \leq \theta \leq 25.00$ & $1.93 \leq \theta \leq 25.00$ & $1.76 \leq \theta \leq 25.00$ & $1.76 \leq \theta \leq 25.00$ \\
\hline \multicolumn{5}{|l|}{ collection. $^{\circ}$} \\
\hline$a, \AA$ & $9.495(3)$ & $13.341(5)$ & $9.063(3)$ & $9.092(6)$ \\
\hline$b, \AA$ & $22.063(7)$ & $11.935(4)$ & $11.951(6)$ & $11.978(9)$ \\
\hline$c, \AA$ & $12.615(4)$ & $9.052(3)$ & $13.357(6)$ & $13.370(10)$ \\
\hline$\alpha,{ }^{\circ}$ & 90 & $101.925(12)$ & $114.54(2)$ & $114.34(2)$ \\
\hline$\beta,{ }^{\circ}$ & 109.932(11) & $100.871(11)$ & $100.74(2)$ & $100.91(2)$ \\
\hline$\gamma,{ }^{\circ}$ & 90 & $114.455(10)$ & $102.03(2)$ & $102.07(2)$ \\
\hline$V, \AA^{3}$ & $2484.6(14)$ & $1221.3(7)$ & $1225.1(9)$ & $1233.7(15)$ \\
\hline$Z$ & 2 & 1 & 1 & 1 \\
\hline$\rho_{\text {calcd }}, \mathrm{g} \mathrm{cm}^{-3}$ & 1.242 & 1.298 & 1.294 & 1.294 \\
\hline$\mu, \mathrm{mm}^{-1}$ & 0.423 & 0.528 & 0.572 & 0.672 \\
\hline GOF & 1.024 & 1.054 & 1.054 & 1.055 \\
\hline$R 1^{\mathrm{a}}$ & 0.0615 & 0.0571 & 0.0570 & 0.0817 \\
\hline$w R 2$ & 0.1978 & 0.1723 & 0.1799 & 0.2533 \\
\hline
\end{tabular}

${ }^{\mathrm{a}} I>2 \sigma(I)$.

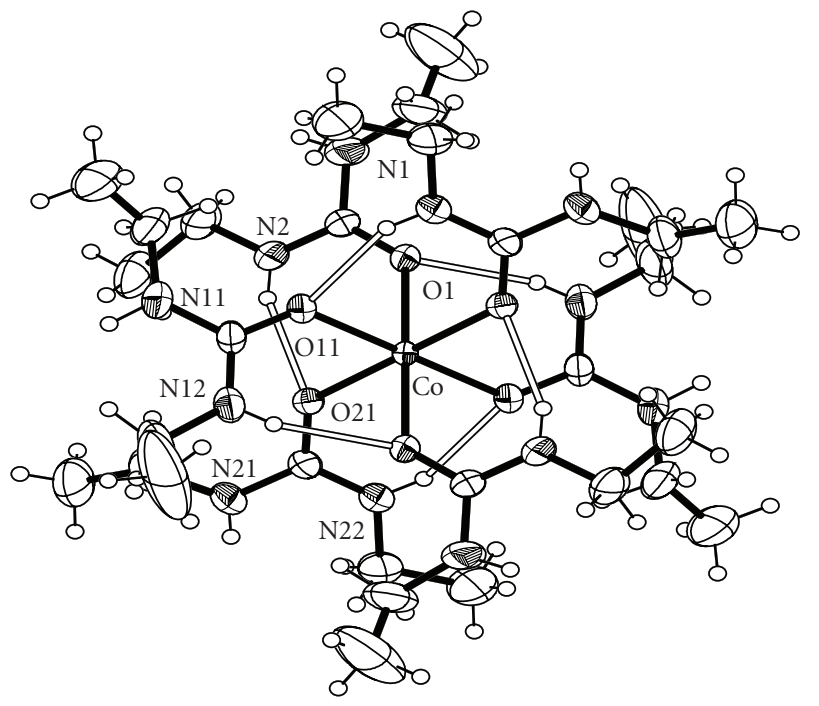

Figure 2: An ORTEP representation of the cation $\left[\mathrm{Co}(\mathrm{DEU})_{6}\right]^{2+}$ present in complex 2 . Open bonds indicate intramolecular hydrogen bonds. The symmetry-equivalent atoms are not labeled.

present in the structure of $\mathbf{1}$. In all four structures, the metal ion sits on an inversion centre and is surrounded by six O-bonded DEU ligands. The $\mathrm{M}-\mathrm{O}_{(\mathrm{DEU})}$ bond distances in 1-4 are comparable to those in $\left[\mathrm{M}(\mathrm{DMU})_{6}\right]^{2+}[17,18]$. The average $\mathrm{M}-\mathrm{O}_{(\mathrm{DEU})}$ bond lengths change according to the sequence $\mathbf{1}[2.098 \AA] \cong \mathbf{2}[2.096 \AA]>\mathbf{3}[2.072 \AA]<\mathbf{4}$ [2.108 $\AA$ ] following the Irving-Williams series [29]. The DEU molecules in $\mathbf{1 - 4}$ are coordinated in a bent fashion forming

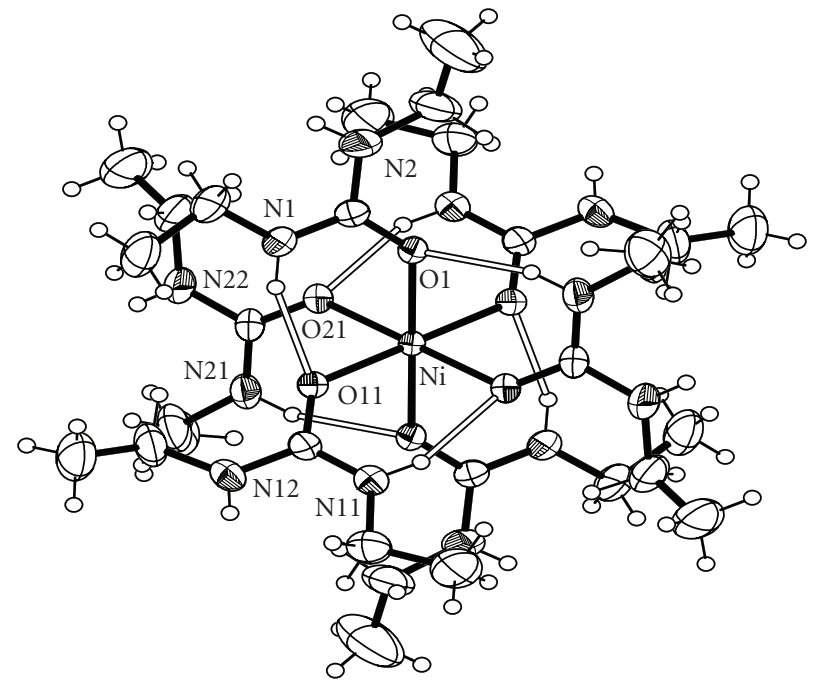

Figure 3: An ORTEP representation of the cation $\left[\mathrm{Ni}(\mathrm{DEU})_{6}\right]^{2+}$ present in complex 3. Open bonds indicate intramolecular hydrogen bonds. The symmetry-equivalent atoms are not labeled.

$\mathrm{C}=\mathrm{O} \cdots \mathrm{M}$ angles ranging from $127.6^{\circ}$ to $132.5^{\circ}$. This is the usual way of coordination of urea and its derivatives and has been observed in the similar $\left[\mathrm{M}(\mathrm{DMU})_{6}\right] \mathrm{X}_{2}$ complexes [1621]. Linearly or approximately linearly coordinated ureas are rare and have been observed only in a few cases [21]. There are six strong intramolecular (intracationic) hydrogen bonds inside each cation with atoms $\mathrm{N}(1), \mathrm{N}(11)$, and $\mathrm{N}(21)$ (and their symmetry equivalents) as donors, and atoms 
TABLE 2: Selected bond lengths $(\AA)$ and angles $\left(^{\circ}\right)$ for $\mathbf{1}$.

\begin{tabular}{|c|c|c|c|}
\hline $\mathrm{Co}-\mathrm{O}(1)$ & $2.094(2)$ & $\mathrm{N}(11)-\mathrm{C}(13)$ & $1.327(5)$ \\
\hline $\mathrm{Co}-\mathrm{O}(11)$ & $2.088(2)$ & $\mathrm{C}(13)-\mathrm{N}(12)$ & $1.323(5)$ \\
\hline $\mathrm{Co}-\mathrm{O}(21)$ & $2.112(2)$ & $\mathrm{N}(12)-\mathrm{C}(14)$ & $1.456(7)$ \\
\hline $\mathrm{O}(1)-\mathrm{C}(3)$ & $1.262(4)$ & $\mathrm{C}(14)-\mathrm{C}(15)$ & $1.417(8)$ \\
\hline $\mathrm{O}(11)-\mathrm{C}(13)$ & $1.267(4)$ & $\mathrm{C}(21)-\mathrm{C}(22)$ & $1.451(8)$ \\
\hline $\mathrm{O}(21)-\mathrm{C}(23)$ & $1.256(4)$ & $\mathrm{C}(22)-\mathrm{N}(21)$ & $1.456(5)$ \\
\hline $\mathrm{C}(1)-\mathrm{C}(2)$ & $1.486(6)$ & $\mathrm{N}(21)-\mathrm{C}(23)$ & $1.325(5)$ \\
\hline $\mathrm{C}(2)-\mathrm{N}(1)$ & $1.450(5)$ & $\mathrm{C}(23)-\mathrm{N}(22)$ & $1.335(5)$ \\
\hline $\mathrm{N}(1)-\mathrm{C}(3)$ & $1.331(5)$ & $\mathrm{N}(22)-\mathrm{C}(24)$ & $1.450(6)$ \\
\hline $\mathrm{C}(3)-\mathrm{N}(2)$ & $1.325(5)$ & $\mathrm{C}(24)-\mathrm{C}(25)$ & $1.390(9)$ \\
\hline $\mathrm{N}(2)-\mathrm{C}(4)$ & $1.462(7)$ & $\mathrm{B}-\mathrm{F}(1)$ & $1.279(6)$ \\
\hline $\mathrm{C}(4)-\mathrm{C}(5)$ & $1.372(9)$ & $\mathrm{B}-\mathrm{F}(2)$ & $1.311(9)$ \\
\hline $\mathrm{C}(11)-\mathrm{C}(12)$ & $1.289(9)$ & $\mathrm{B}-\mathrm{F}(3)$ & $1.331(8)$ \\
\hline $\mathrm{C}(12)-\mathrm{N}(11)$ & $1.459(6)$ & $\mathrm{B}-\mathrm{F}(4)$ & $1.265(10)$ \\
\hline $\mathrm{O}(11) \# 1-\mathrm{Co}-\mathrm{O}(11)$ & $180.00(19)$ & $\mathrm{C}(3)-\mathrm{N}(2)-\mathrm{C}(4)$ & $124.0(4)$ \\
\hline $\mathrm{O}(11) \# 1-\mathrm{Co}-\mathrm{O}(1)$ & $92.93(10)$ & $\mathrm{C}(5)-\mathrm{C}(4)-\mathrm{N}(2)$ & $111.7(6)$ \\
\hline $\mathrm{O}(11)-\mathrm{Co}-\mathrm{O}(1)$ & $87.07(10)$ & $\mathrm{C}(11)-\mathrm{C}(12)-\mathrm{N}(11)$ & $119.0(7)$ \\
\hline $\mathrm{O}(11) \# 1-\mathrm{Co}-\mathrm{O}(1) \# 1$ & $87.07(10)$ & $\mathrm{C}(13)-\mathrm{N}(11)-\mathrm{C}(12)$ & $126.9(4)$ \\
\hline $\mathrm{O}(11)-\mathrm{Co}-\mathrm{O}(1) \# 1$ & $92.93(10)$ & $\mathrm{O}(11)-\mathrm{C}(13)-\mathrm{N}(12)$ & $119.6(4)$ \\
\hline $\mathrm{O}(1)-\mathrm{Co}-\mathrm{O}(1) \# 1$ & $180.00(8)$ & $\mathrm{O}(11)-\mathrm{C}(13)-\mathrm{N}(11)$ & $120.4(3)$ \\
\hline $\mathrm{O}(11) \# 1-\mathrm{Co}-\mathrm{O}(21) \# 1$ & $86.69(10)$ & $\mathrm{N}(12)-\mathrm{C}(13)-\mathrm{N}(11)$ & $120.0(4)$ \\
\hline $\mathrm{O}(11)-\mathrm{Co}-\mathrm{O}(21) \# 1$ & $93.31(10)$ & $\mathrm{C}(13)-\mathrm{N}(12)-\mathrm{C}(14)$ & $125.1(4)$ \\
\hline $\mathrm{O}(1)-\mathrm{Co}-\mathrm{O}(21) \# 1$ & $87.20(10)$ & $\mathrm{C}(15)-\mathrm{C}(14)-\mathrm{N}(12)$ & $112.8(6)$ \\
\hline $\mathrm{O}(1) \# 1-\mathrm{Co}-\mathrm{O}(21) \# 1$ & $92.80(10)$ & $\mathrm{C}(21)-\mathrm{C}(22)-\mathrm{N}(21)$ & $113.9(5)$ \\
\hline $\mathrm{O}(11) \# 1-\mathrm{Co}-\mathrm{O}(21)$ & $93.31(10)$ & $\mathrm{C}(23)-\mathrm{N}(21)-\mathrm{C}(22)$ & $127.9(4)$ \\
\hline $\mathrm{O}(11)-\mathrm{Co}-\mathrm{O}(21)$ & $86.69(10)$ & $\mathrm{O}(21)-\mathrm{C}(23)-\mathrm{N}(21)$ & $121.0(3)$ \\
\hline $\mathrm{O}(1)-\mathrm{Co}-\mathrm{O}(21)$ & $92.80(10)$ & $\mathrm{O}(21)-\mathrm{C}(23)-\mathrm{N}(22)$ & $120.1(3)$ \\
\hline $\mathrm{O}(1) \# 1-\mathrm{Co}-\mathrm{O}(21)$ & $87.20(10)$ & $\mathrm{N}(21)-\mathrm{C}(23)-\mathrm{N}(22)$ & $119.0(3)$ \\
\hline $\mathrm{O}(21) \# 1-\mathrm{Co}-\mathrm{O}(21)$ & $180.00(12)$ & $\mathrm{C}(23)-\mathrm{N}(22)-\mathrm{C}(24)$ & $124.3(4)$ \\
\hline $\mathrm{C}(3)-\mathrm{O}(1)-\mathrm{Co}$ & $132.5(2)$ & $\mathrm{C}(25)-\mathrm{C}(24)-\mathrm{N}(22)$ & 114.1(6) \\
\hline $\mathrm{C}(13)-\mathrm{O}(11)-\mathrm{Co}$ & $127.6(2)$ & $\mathrm{F}(4)-\mathrm{B}-\mathrm{F}(1)$ & 111.2(9) \\
\hline $\mathrm{C}(23)-\mathrm{O}(21)-\mathrm{Co}$ & $129.7(2)$ & $\mathrm{F}(4)-\mathrm{B}-\mathrm{F}(2)$ & $103.0(7)$ \\
\hline $\mathrm{N}(1)-\mathrm{C}(2)-\mathrm{C}(1)$ & $111.0(4)$ & $\mathrm{F}(1)-\mathrm{B}-\mathrm{F}(2)$ & $115.0(6)$ \\
\hline $\mathrm{C}(3)-\mathrm{N}(1)-\mathrm{C}(2)$ & $125.8(3)$ & $\mathrm{F}(4)-\mathrm{B}-\mathrm{F}(3)$ & $105.1(8)$ \\
\hline $\mathrm{O}(1)-\mathrm{C}(3)-\mathrm{N}(2)$ & $119.9(4)$ & $\mathrm{F}(1)-\mathrm{B}-\mathrm{F}(3)$ & $115.1(5)$ \\
\hline $\mathrm{O}(1)-\mathrm{C}(3)-\mathrm{N}(1)$ & $121.2(3)$ & $\mathrm{F}(2)-\mathrm{B}-\mathrm{F}(3)$ & $106.3(7)$ \\
\hline $\mathrm{N}(2)-\mathrm{C}(3)-\mathrm{N}(1)$ & $118.9(4)$ & & \\
\hline
\end{tabular}

Symmetry transformation used to generate equivalent atoms: $\# 1-x,-y,-z$.

$\mathrm{O}(1), \mathrm{O}(11)$ and $\mathrm{O}(21)$ (and their symmetry equivalents) as acceptors for $\mathbf{1}, \mathbf{3}$ and $\mathbf{4}$ and $\mathrm{N}(2), \mathrm{N}(12)$ and $\mathrm{N}(22)$ (and their symmetry equivalents) as donors, and atoms $\mathrm{O}(1), \mathrm{O}(11)$ and $\mathrm{O}(21)$ (and their symmetry equivalents) as acceptors for 2. These intracationic hydrogen bonds create six-membered pseudochelate rings providing extra stabilization to the $\left[\mathrm{M}(\mathrm{DEU})_{6}\right]^{2+}$ cation. Overall the structural characteristics, that is, bond distances, agnles and intracation hydrogen bonding interactions in the $\left[\mathrm{M}(\mathrm{DEU})_{6}\right]^{2+}$ resemble those found in the $\left[\mathrm{M}(\mathrm{DMU})_{6}\right]^{2+}$ cations $[17,18]$ with an exception regarding two additional $\mathrm{C}-\mathrm{H} \cdots \mathrm{O} \mathrm{H}$-bonds (and their symmetry equivalent) found in $\left[\mathrm{Co}(\mathrm{DMU})_{6}\right]\left(\mathrm{ClO}_{4}\right)_{2}$ and $\left[\mathrm{Co}(\mathrm{DMU})_{6}\right]\left(\mathrm{BF}_{4}\right)_{2}$ [18]. Complexes 1-4 extend to seven the number of structurally characterised DEU compounds. The three, previously structurally characterised, compounds are $\left[\mathrm{SnBr}_{4}(\mathrm{DEU})_{2}\right][22],\left[\mathrm{Fe}(\mathrm{DEU})_{6}\right]\left(\mathrm{ClO}_{4}\right)_{2}$ [23] and $\left[\mathrm{Mn}(\mathrm{DEU})_{6}\right]\left[\mathrm{MnBr}_{4}\right]$ [24]. Complexes 2-4 are isostructural to $\left[\mathrm{Fe}(\mathrm{DEU})_{6}\right]\left(\mathrm{ClO}_{4}\right)_{2}$. The average $\mathrm{Fe}-\mathrm{O}_{\mathrm{DEU}}$ bond distance is 2.105 following the Irving-Williams series as stated above.

Although the intracationic H-bonding interactions are the same along the $\left[\mathrm{M}(\mathrm{DEU})_{6}\right]^{2+}$ series as well as very similar with those found in the $\left[\mathrm{M}(\mathrm{DMU})_{6}\right]^{2+}$ cations, the intermolecular/interionic interactions are quite different. That the complexes $\mathbf{2 - 4}$ are isostructural implies that the interionic hydrogen bonding interactions are the same. 
TABLe 3: Selected bond lengths $(\AA)$ and angles $\left({ }^{\circ}\right)$ for 2 .

\begin{tabular}{|c|c|c|c|}
\hline $\mathrm{Co}-\mathrm{O}(1)$ & $2.090(2)$ & $\mathrm{N}(11)-\mathrm{C}(13)$ & $1.329(4)$ \\
\hline $\mathrm{Co}-\mathrm{O}(11)$ & $2.100(2)$ & $\mathrm{C}(13)-\mathrm{N}(12)$ & $1.323(5)$ \\
\hline $\mathrm{Co}-\mathrm{O}(21)$ & $2.097(2)$ & $\mathrm{N}(12)-\mathrm{C}(14)$ & $1.446(5)$ \\
\hline $\mathrm{O}(1)-\mathrm{C}(3)$ & $1.259(4)$ & $C(14)-C(15)$ & $1.213(9)$ \\
\hline $\mathrm{O}(11)-\mathrm{C}(13)$ & $1.260(4)$ & $\mathrm{C}(21)-\mathrm{C}(22)$ & $1.424(7)$ \\
\hline $\mathrm{O}(21)-\mathrm{C}(23)$ & $1.265(4)$ & $\mathrm{C}(22)-\mathrm{N}(21)$ & $1.456(6)$ \\
\hline$C(1)-C(2)$ & $1.329(8)$ & $\mathrm{N}(21)-\mathrm{C}(23)$ & $1.330(4)$ \\
\hline $\mathrm{C}(2)-\mathrm{N}(1)$ & $1.455(6)$ & $\mathrm{C}(23)-\mathrm{N}(22)$ & $1.324(4)$ \\
\hline $\mathrm{N}(1)-\mathrm{C}(3)$ & $1.329(4)$ & $\mathrm{N}(22)-\mathrm{C}(24)$ & $1.457(5)$ \\
\hline $\mathrm{C}(3)-\mathrm{N}(2)$ & $1.328(4)$ & $\mathrm{C}(24)-\mathrm{C}(25)$ & $1.489(7)$ \\
\hline$N(2)-C(4)$ & $1.455(4)$ & $\mathrm{Cl}-\mathrm{O}(34)$ & $1.257(8)$ \\
\hline$C(4)-C(5)$ & $1.488(6)$ & $\mathrm{Cl}-\mathrm{O}(32)$ & $1.295(5)$ \\
\hline $\mathrm{C}(11)-\mathrm{C}(12)$ & $1.456(7)$ & $\mathrm{Cl}-\mathrm{O}(31)$ & $1.307(5)$ \\
\hline $\mathrm{C}(12)-\mathrm{N}(11)$ & $1.455(6)$ & $\mathrm{Cl}-\mathrm{O}(33)$ & $1.386(8)$ \\
\hline $\mathrm{O}(1) \# 1-\mathrm{Co}-\mathrm{O}(1)$ & $180.00(9)$ & $\mathrm{C}(3)-\mathrm{N}(2)-\mathrm{C}(4)$ & $125.7(3)$ \\
\hline $\mathrm{O}(1) \# 1-\mathrm{Co}-\mathrm{O}(21)$ & $86.56(9)$ & $\mathrm{N}(2)-\mathrm{C}(4)-\mathrm{C}(5)$ & $110.8(4)$ \\
\hline $\mathrm{O}(1)-\mathrm{Co}-\mathrm{O}(21)$ & $93.44(9)$ & $\mathrm{N}(11)-\mathrm{C}(12)-\mathrm{C}(11)$ & $112.4(4)$ \\
\hline $\mathrm{O}(1) \# 1-\mathrm{Co}-\mathrm{O}(21) \# 1$ & $93.44(9)$ & $\mathrm{C}(13)-\mathrm{N}(11)-\mathrm{C}(12)$ & $123.5(3)$ \\
\hline $\mathrm{O}(1)-\mathrm{Co}-\mathrm{O}(21) \# 1$ & $86.56(9)$ & $\mathrm{O}(11)-\mathrm{C}(13)-\mathrm{N}(12)$ & $121.2(3)$ \\
\hline $\mathrm{O}(21)-\mathrm{Co}-\mathrm{O}(21) \# 1$ & $180.00(16)$ & $\mathrm{O}(11)-\mathrm{C}(13)-\mathrm{N}(11)$ & $120.4(3)$ \\
\hline $\mathrm{O}(1) \# 1-\mathrm{Co}-\mathrm{O}(11)$ & $93.24(9)$ & $\mathrm{N}(12)-\mathrm{C}(13)-\mathrm{N}(11)$ & $118.4(3)$ \\
\hline $\mathrm{O}(1)-\mathrm{Co}-\mathrm{O}(11)$ & $86.76(9)$ & $\mathrm{C}(13)-\mathrm{N}(12)-\mathrm{C}(14)$ & $127.8(4)$ \\
\hline $\mathrm{O}(21)-\mathrm{Co}-\mathrm{O}(11)$ & $86.22(8)$ & $\mathrm{C}(15)-\mathrm{C}(14)-\mathrm{N}(12)$ & $120.2(5)$ \\
\hline $\mathrm{O}(21) \# 1-\mathrm{Co}-\mathrm{O}(11)$ & $93.78(8)$ & $\mathrm{C}(21)-\mathrm{C}(22)-\mathrm{N}(21)$ & $113.4(5)$ \\
\hline $\mathrm{O}(1) \# 1-\mathrm{Co}-\mathrm{O}(11) \# 1$ & $86.76(9)$ & $\mathrm{C}(23)-\mathrm{N}(21)-\mathrm{C}(22)$ & $123.9(3)$ \\
\hline $\mathrm{O}(1)-\mathrm{Co}-\mathrm{O}(11) \# 1$ & $93.24(9)$ & $\mathrm{O}(21)-\mathrm{C}(23)-\mathrm{N}(22)$ & $121.1(3)$ \\
\hline $\mathrm{O}(21)-\mathrm{Co}-\mathrm{O}(11) \# 1$ & $93.78(8)$ & $\mathrm{O}(21)-\mathrm{C}(23)-\mathrm{N}(21)$ & $120.3(3)$ \\
\hline $\mathrm{O}(21) \# 1-\mathrm{Co}-\mathrm{O}(11) \# 1$ & $86.22(8)$ & $\mathrm{N}(22)-\mathrm{C}(23)-\mathrm{N}(21)$ & $118.6(3)$ \\
\hline $\mathrm{O}(11)-\mathrm{Co}-\mathrm{O}(11) \# 1$ & $180.00(11)$ & $\mathrm{C}(23)-\mathrm{N}(22)-\mathrm{C}(24)$ & $126.2(3)$ \\
\hline $\mathrm{C}(3)-\mathrm{O}(1)-\mathrm{Co}$ & $129.82(19)$ & $\mathrm{N}(22)-\mathrm{C}(24)-\mathrm{C}(25)$ & $110.0(4)$ \\
\hline $\mathrm{C}(13)-\mathrm{O}(11)-\mathrm{Co}$ & $129.3(2)$ & $\mathrm{O}(34)-\mathrm{Cl}-\mathrm{O}(32)$ & $114.5(8)$ \\
\hline $\mathrm{C}(23)-\mathrm{O}(21)-\mathrm{Co}$ & $129.46(18)$ & $\mathrm{O}(34)-\mathrm{Cl}-\mathrm{O}(31)$ & $119.6(7)$ \\
\hline $\mathrm{C}(1)-\mathrm{C}(2)-\mathrm{N}(1)$ & $115.3(6)$ & $\mathrm{O}(32)-\mathrm{Cl}-\mathrm{O}(31)$ & $112.1(5)$ \\
\hline $\mathrm{C}(3)-\mathrm{N}(1)-\mathrm{C}(2)$ & $123.4(3)$ & $\mathrm{O}(34)-\mathrm{Cl}-\mathrm{O}(33)$ & $98.7(9)$ \\
\hline $\mathrm{O}(1)-\mathrm{C}(3)-\mathrm{N}(2)$ & $121.6(3)$ & $\mathrm{O}(32)-\mathrm{Cl}-\mathrm{O}(33)$ & $105.0(7)$ \\
\hline $\mathrm{O}(1)-\mathrm{C}(3)-\mathrm{N}(1)$ & $119.8(3)$ & $\mathrm{O}(31)-\mathrm{Cl}-\mathrm{O}(33)$ & $104.2(6)$ \\
\hline $\mathrm{N}(2)-\mathrm{C}(3)-\mathrm{N}(1)$ & $118.5(3)$ & & \\
\hline
\end{tabular}

Symmetry transformation used to generate equivalent atoms: $\# 1-x,-y,-z$.

Therefore, only the hydrogen bonding of the representative complex 4 will be discussed. The $\left[\mathrm{Zn}(\mathrm{DEU})_{6}\right]^{2+}$ and $\mathrm{ClO}_{4}{ }^{-}$ ions in 4 have assembled to create an infinite 2D network through three crystallographically independent inter-

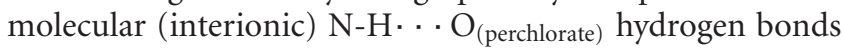
(and their symmetry related) (Figure 5). Each perchlorate accepts three hydrogen bonds with the $\mathrm{O}(31), \mathrm{O}(32)$, and $\mathrm{O}(33)$ atoms acting as hydrogen bond acceptors while each $\left[\mathrm{Zn}(\mathrm{DEU})_{6}\right]^{2+}$ connects to six $\mathrm{ClO}_{4}{ }^{-}$anions through the remaining $\mathrm{N}-\mathrm{H}$ groups (Figure 5). As a consequence of the participation of $\mathrm{O}(31), \mathrm{O}(32)$, and $\mathrm{O}(33)$ in hydrogen bonding, the $\mathrm{Cl}-\mathrm{O}(31), \mathrm{Cl}-\mathrm{O}(32)$, and $\mathrm{Cl}-\mathrm{O}(33)$ bond lengths [1.402(1), 1.318(1) and 1.440(1) $\AA$, resp.] are slightly longer than the $\mathrm{Cl}-\mathrm{O}(34)[1.290(1) \AA]$. In this arrangement, a binodal $(3,6)$-connected network forms with Schläfli symbol $\left(4^{3}\right)_{2}\left(4^{6} .6^{6} \cdot 8^{3}\right)$ (Figure 6). This two-dimensional (2D) hydrogen-bonded kgd net is the dual of the kagome kgm(3.6.3.6) net. It is worth noting that the $2 \mathrm{D}$ network adopted by $2-4$ was not adopted by any of the $\left[\mathrm{M}(\mathrm{DMU})_{6}\right]\left(\mathrm{ClO}_{4}\right)_{2}$ complexes $[17,18]$ suggesting that the substitution of DMU by DEU substantially changes the intermolecular (interionic) interactions probably due to the larger ethyl groups (in DEU) instead of the smaller methyl groups (in DMU). Similar 2D networks have been adopted by $\left[\mathrm{Zn}(\mathrm{DMU})_{6}\right]\left(\mathrm{ClO}_{4}\right)_{2}$ [17] and $\left[\mathrm{Co}(\mathrm{DMU})_{6}\right]\left(\mathrm{BF}_{4}\right)_{2}[18]$ with the $\mathrm{ClO}_{4}{ }^{-}$and the $\mathrm{BF}_{4}{ }^{-}$ anions acting as 3 -connected nodes and the $\left[\mathrm{M}(\mathrm{DMU})_{6}\right]^{2+}$ 
TABLE 4: Selected bond lengths $(\AA)$ and angles $\left(^{\circ}\right)$ for 3 .

\begin{tabular}{|c|c|c|c|}
\hline $\mathrm{Ni}-\mathrm{O}(1)$ & $2.068(2)$ & $\mathrm{N}(11)-\mathrm{C}(13)$ & $1.323(5)$ \\
\hline $\mathrm{Ni}-\mathrm{O}(11)$ & $2.073(2)$ & $\mathrm{C}(13)-\mathrm{N}(12)$ & $1.334(5)$ \\
\hline $\mathrm{Ni}-\mathrm{O}(21)$ & $2.076(2)$ & $\mathrm{N}(12)-\mathrm{C}(14)$ & $1.461(6)$ \\
\hline $\mathrm{O}(1)-\mathrm{C}(3)$ & $1.257(4)$ & $\mathrm{C}(14)-\mathrm{C}(15)$ & $1.415(8)$ \\
\hline $\mathrm{O}(11)-\mathrm{C}(13)$ & $1.266(4)$ & $\mathrm{C}(21)-\mathrm{C}(22)$ & $1.337(16)$ \\
\hline $\mathrm{O}(21)-\mathrm{C}(23)$ & $1.263(4)$ & $\mathrm{C}(22)-\mathrm{N}(21)$ & $1.460(5)$ \\
\hline$C(1)-C(2)$ & $1.498(7)$ & $\mathrm{N}(21)-\mathrm{C}(23)$ & $1.322(5)$ \\
\hline $\mathrm{C}(2)-\mathrm{N}(1)$ & $1.462(5)$ & $\mathrm{C}(23)-\mathrm{N}(22)$ & $1.335(5)$ \\
\hline $\mathrm{N}(1)-\mathrm{C}(3)$ & $1.329(5)$ & $\mathrm{N}(22)-\mathrm{C}(24)$ & $1.454(6)$ \\
\hline $\mathrm{C}(3)-\mathrm{N}(2)$ & $1.336(5)$ & $C(24)-C(25)$ & $1.462(8)$ \\
\hline $\mathrm{N}(2)-\mathrm{C}(4)$ & $1.458(7)$ & $\mathrm{Cl}-\mathrm{O}(34)$ & $1.274(8)$ \\
\hline$C(4)-C(5)$ & $1.368(9)$ & $\mathrm{Cl}-\mathrm{O}(32)$ & $1.298(5)$ \\
\hline$C(11)-C(12)$ & $1.488(7)$ & $\mathrm{Cl}-\mathrm{O}(31)$ & $1.310(6)$ \\
\hline $\mathrm{C}(12)-\mathrm{N}(11)$ & $1.459(5)$ & $\mathrm{Cl}-\mathrm{O}(33)$ & $1.378(9)$ \\
\hline $\mathrm{O}(1) \# 1-\mathrm{Ni}-\mathrm{O}(1)$ & $180.00(11)$ & $\mathrm{C}(3)-\mathrm{N}(2)-\mathrm{C}(4)$ & $123.0(4)$ \\
\hline $\mathrm{O}(1) \# 1-\mathrm{Ni}-\mathrm{O}(11)$ & $86.68(10)$ & $\mathrm{C}(5)-\mathrm{C}(4)-\mathrm{N}(2)$ & $114.0(6)$ \\
\hline $\mathrm{O}(1)-\mathrm{Ni}-\mathrm{O}(11)$ & $93.32(10)$ & $\mathrm{N}(11)-\mathrm{C}(12)-\mathrm{C}(11)$ & $110.5(4)$ \\
\hline $\mathrm{O}(1) \# 1-\mathrm{Ni}-\mathrm{O}(11) \# 1$ & $93.32(10)$ & $\mathrm{C}(13)-\mathrm{N}(11)-\mathrm{C}(12)$ & $126.5(3)$ \\
\hline $\mathrm{O}(1)-\mathrm{Ni}-\mathrm{O}(11) \# 1$ & $86.68(10)$ & $\mathrm{O}(11)-\mathrm{C}(13)-\mathrm{N}(11)$ & $121.6(3)$ \\
\hline $\mathrm{O}(11)-\mathrm{Ni}-\mathrm{O}(11) \# 1$ & $180.00(17)$ & $\mathrm{O}(11)-\mathrm{C}(13)-\mathrm{N}(12)$ & $120.0(3)$ \\
\hline $\mathrm{O}(1) \# 1-\mathrm{Ni}-\mathrm{O}(21) \# 1$ & $86.72(10)$ & $\mathrm{N}(11)-\mathrm{C}(13)-\mathrm{N}(12)$ & $118.4(3)$ \\
\hline $\mathrm{O}(1)-\mathrm{Ni}-\mathrm{O}(21) \# 1$ & $93.28(10)$ & $C(13)-N(12)-C(14)$ & $123.8(4)$ \\
\hline $\mathrm{O}(11)-\mathrm{Ni}-\mathrm{O}(21) \# 1$ & $93.76(10)$ & $\mathrm{C}(15)-\mathrm{C}(14)-\mathrm{N}(12)$ & $113.9(5)$ \\
\hline $\mathrm{O}(11) \# 1-\mathrm{Ni}-\mathrm{O}(21) \# 1$ & $86.24(10)$ & $\mathrm{C}(21)-\mathrm{C}(22)-\mathrm{N}(21)$ & $117.1(9)$ \\
\hline $\mathrm{O}(1) \# 1-\mathrm{Ni}-\mathrm{O}(21)$ & $93.28(10)$ & $\mathrm{C}(23)-\mathrm{N}(21)-\mathrm{C}(22)$ & $128.0(4)$ \\
\hline $\mathrm{O}(1)-\mathrm{Ni}-\mathrm{O}(21)$ & $86.72(10)$ & $\mathrm{O}(21)-\mathrm{C}(23)-\mathrm{N}(21)$ & $121.5(3)$ \\
\hline $\mathrm{O}(11)-\mathrm{Ni}-\mathrm{O}(21)$ & $86.24(10)$ & $\mathrm{O}(21)-\mathrm{C}(23)-\mathrm{N}(22)$ & $119.7(4)$ \\
\hline $\mathrm{O}(11) \# 1-\mathrm{Ni}-\mathrm{O}(21)$ & $93.76(10)$ & $\mathrm{N}(21)-\mathrm{C}(23)-\mathrm{N}(22)$ & $118.8(3)$ \\
\hline $\mathrm{O}(21) \# 1-\mathrm{Ni}-\mathrm{O}(21)$ & $180.00(13)$ & $\mathrm{C}(23)-\mathrm{N}(22)-\mathrm{C}(24)$ & $123.8(4)$ \\
\hline $\mathrm{C}(3)-\mathrm{O}(1)-\mathrm{Ni}$ & $130.2(2)$ & $\mathrm{N}(22)-\mathrm{C}(24)-\mathrm{C}(25)$ & $112.7(5)$ \\
\hline $\mathrm{C}(13)-\mathrm{O}(11)-\mathrm{Ni}$ & $129.4(2)$ & $\mathrm{O}(34)-\mathrm{Cl}-\mathrm{O}(32)$ & $113.6(8)$ \\
\hline $\mathrm{C}(23)-\mathrm{O}(21)-\mathrm{Ni}$ & $129.5(2)$ & $\mathrm{O}(34)-\mathrm{Cl}-\mathrm{O}(31)$ & $118.5(8)$ \\
\hline $\mathrm{N}(1)-\mathrm{C}(2)-\mathrm{C}(1)$ & $110.3(4)$ & $\mathrm{O}(32)-\mathrm{Cl}-\mathrm{O}(31)$ & $113.0(5)$ \\
\hline $\mathrm{C}(3)-\mathrm{N}(1)-\mathrm{C}(2)$ & $125.5(3)$ & $\mathrm{O}(34)-\mathrm{Cl}-\mathrm{O}(33)$ & $98.6(10)$ \\
\hline $\mathrm{O}(1)-\mathrm{C}(3)-\mathrm{N}(1)$ & $121.9(3)$ & $\mathrm{O}(32)-\mathrm{Cl}-\mathrm{O}(33)$ & $105.1(7)$ \\
\hline $\mathrm{O}(1)-\mathrm{C}(3)-\mathrm{N}(2)$ & $119.8(3)$ & $\mathrm{O}(31)-\mathrm{Cl}-\mathrm{O}(33)$ & $105.7(7)$ \\
\hline $\mathrm{N}(1)-\mathrm{C}(3)-\mathrm{N}(2)$ & $118.3(3)$ & & \\
\hline
\end{tabular}

Symmetry transformation used to generate equivalent atoms: \#1 $-x,-y,-z$.

acting as 6-connected nodes but the connections are achieved through two $\mathrm{N}-\mathrm{H} \cdot \cdots \mathrm{X}$ and one $\mathrm{C}-\mathrm{H} \cdot \cdots \mathrm{X}$ hydrogen bonds (and their symmetry equivalents), $\left(\mathrm{X}=\mathrm{O}_{\text {(perchlorate) }}\right.$ or $\mathrm{F}$ (tetrafluoroborate), resp.).

The intermolecular hydrogen bonding interactions in $\mathbf{1}$ are far more interesting that those in $2-4$. The $\left[\mathrm{Co}(\mathrm{DEU})_{6}\right]^{2+}$ and the $\mathrm{BF}_{4}{ }^{-}$anions have assembled to create a threedimensional (3D) hydrogen-bonded framework through three crystallographically independent intermolecular (interionic) N-H . - F (tetrafluoroborate) hydrogen bonds (and their symmetry equivalents). Each $\mathrm{BF}_{4}^{-}$accepts three hydrogen bonds with the $\mathrm{F}(1), \mathrm{F}(2)$ and $\mathrm{F}(3)$ atoms acting as hydrogen bond acceptors while each $\left[\mathrm{Co}(\mathrm{DEU})_{6}\right]^{2+}$ connects to six $\mathrm{BF}_{4}{ }^{-}$anions through the remaining $\mathrm{N}-\mathrm{H}$ groups (Figure 7 ). In this arrangement, a $(3,6)$-connected network forms with the $\left[\mathrm{Co}(\mathrm{DEU})_{6}\right]^{2+}$ cations acting as the 6-connected nodes and the $\mathrm{BF}_{4}{ }^{-}$anions as the 3 -connected nodes. Although the connectivity of each ion seems identical to that found in $2-4$, the arrangement of the $\left[\mathrm{Co}(\mathrm{DEU})_{6}\right]^{2+}$ and $\mathrm{BF}_{4}{ }^{-}$ions is quite different resulting in a binodal $3 \mathrm{D}$ hydrogen-bonded 
TABLE 5: Selected bond lengths $(\AA)$ and angles $\left(^{\circ}\right)$ for 4 .

\begin{tabular}{|c|c|c|c|}
\hline $\mathrm{Zn}-\mathrm{O}(1)$ & $2.108(3)$ & $\mathrm{N}(11)-\mathrm{C}(13)$ & $1.330(6)$ \\
\hline $\mathrm{Zn}-\mathrm{O}(11)$ & $2.107(3)$ & $\mathrm{C}(13)-\mathrm{N}(12)$ & $1.328(6)$ \\
\hline $\mathrm{Zn}-\mathrm{O}(21)$ & $2.111(3)$ & $\mathrm{N}(12)-\mathrm{C}(14)$ & $1.470(9)$ \\
\hline $\mathrm{O}(1)-\mathrm{C}(3)$ & $1.265(5)$ & $\mathrm{C}(14)-\mathrm{C}(15)$ & $1.313(12)$ \\
\hline $\mathrm{O}(11)-\mathrm{C}(13)$ & $1.263(5)$ & $\mathrm{C}(21)-\mathrm{C}(22)$ & $1.208(14)$ \\
\hline $\mathrm{O}(21)-\mathrm{C}(23)$ & $1.279(5)$ & $\mathrm{C}(22)-\mathrm{N}(21)$ & $1.469(7)$ \\
\hline$C(1)-C(2)$ & $1.485(10)$ & $\mathrm{N}(21)-\mathrm{C}(23)$ & $1.315(7)$ \\
\hline $\mathrm{C}(2)-\mathrm{N}(1)$ & $1.457(6)$ & $\mathrm{C}(23)-\mathrm{N}(22)$ & $1.328(6)$ \\
\hline $\mathrm{N}(1)-\mathrm{C}(3)$ & $1.331(6)$ & $\mathrm{N}(22)-\mathrm{C}(24)$ & $1.437(9)$ \\
\hline $\mathrm{C}(3)-\mathrm{N}(2)$ & $1.337(6)$ & $\mathrm{C}(24)-\mathrm{C}(25)$ & $1.496(10)$ \\
\hline $\mathrm{N}(2)-\mathrm{C}(4)$ & $1.452(8)$ & $\mathrm{Cl}-\mathrm{O}(34)$ & $1.29(2)$ \\
\hline$C(4)-C(5)$ & $1.431(10)$ & $\mathrm{Cl}-\mathrm{O}(32)$ & $1.318(12)$ \\
\hline $\mathrm{C}(11)-\mathrm{C}(12)$ & $1.510(9)$ & $\mathrm{Cl}-\mathrm{O}(31)$ & $1.402(13)$ \\
\hline $\mathrm{C}(12)-\mathrm{N}(11)$ & $1.461(6)$ & $\mathrm{Cl}-\mathrm{O}(33)$ & $1.440(16)$ \\
\hline $\mathrm{O}(11) \# 1-\mathrm{Zn}-\mathrm{O}(11)$ & $180.0(2)$ & $\mathrm{C}(3)-\mathrm{N}(2)-\mathrm{C}(4)$ & $124.0(5)$ \\
\hline $\mathrm{O}(11) \# 1-\mathrm{Zn}-\mathrm{O}(1) \# 1$ & $92.91(13)$ & $\mathrm{C}(5)-\mathrm{C}(4)-\mathrm{N}(2)$ & $113.5(7)$ \\
\hline $\mathrm{O}(11)-\mathrm{Zn}-\mathrm{O}(1) \# 1$ & $87.09(13)$ & $\mathrm{N}(11)-\mathrm{C}(12)-\mathrm{C}(11)$ & $109.7(5)$ \\
\hline $\mathrm{O}(11) \# 1-\mathrm{Zn}-\mathrm{O}(1)$ & $87.09(13)$ & $\mathrm{C}(13)-\mathrm{N}(11)-\mathrm{C}(12)$ & $124.8(4)$ \\
\hline $\mathrm{O}(11)-\mathrm{Zn}-\mathrm{O}(1)$ & $92.91(13)$ & $\mathrm{O}(11)-\mathrm{C}(13)-\mathrm{N}(12)$ & $119.8(4)$ \\
\hline $\mathrm{O}(1) \# 1-\mathrm{Zn}-\mathrm{O}(1)$ & 180.00 & $\mathrm{O}(11)-\mathrm{C}(13)-\mathrm{N}(11)$ & $121.5(4)$ \\
\hline $\mathrm{O}(11) \# 1-\mathrm{Zn}-\mathrm{O}(21)$ & $92.83(12)$ & $\mathrm{N}(12)-\mathrm{C}(13)-\mathrm{N}(11)$ & $118.7(4)$ \\
\hline $\mathrm{O}(11)-\mathrm{Zn}-\mathrm{O}(21)$ & $87.17(12)$ & $\mathrm{C}(13)-\mathrm{N}(12)-\mathrm{C}(14)$ & $123.6(5)$ \\
\hline $\mathrm{O}(1) \# 1-\mathrm{Zn}-\mathrm{O}(21)$ & $93.04(12)$ & $\mathrm{C}(15)-\mathrm{C}(14)-\mathrm{N}(12)$ & $116.1(8)$ \\
\hline $\mathrm{O}(1)-\mathrm{Zn}-\mathrm{O}(21)$ & $86.96(12)$ & $\mathrm{C}(21)-\mathrm{C}(22)-\mathrm{N}(21)$ & $121.2(8)$ \\
\hline $\mathrm{O}(11) \# 1-\mathrm{Zn}-\mathrm{O}(21) \# 1$ & $87.17(12)$ & $\mathrm{C}(23)-\mathrm{N}(21)-\mathrm{C}(22)$ & $128.4(5)$ \\
\hline $\mathrm{O}(11)-\mathrm{Zn}-\mathrm{O}(21) \# 1$ & $92.83(12)$ & $\mathrm{O}(21)-\mathrm{C}(23)-\mathrm{N}(21)$ & $121.3(4)$ \\
\hline $\mathrm{O}(1) \# 1-\mathrm{Zn}-\mathrm{O}(21) \# 1$ & $86.96(12)$ & $\mathrm{O}(21)-\mathrm{C}(23)-\mathrm{N}(22)$ & $119.2(5)$ \\
\hline $\mathrm{O}(1)-\mathrm{Zn}-\mathrm{O}(21) \# 1$ & $93.04(12)$ & $\mathrm{N}(21)-\mathrm{C}(23)-\mathrm{N}(22)$ & $119.4(4)$ \\
\hline $\mathrm{O}(21)-\mathrm{Zn}-\mathrm{O}(21) \# 1$ & $180.00(18)$ & $\mathrm{C}(23)-\mathrm{N}(22)-\mathrm{C}(24)$ & $124.9(5)$ \\
\hline $\mathrm{C}(3)-\mathrm{O}(1)-\mathrm{Zn}$ & $129.5(3)$ & $\mathrm{N}(22)-\mathrm{C}(24)-\mathrm{C}(25)$ & $112.6(7)$ \\
\hline $\mathrm{C}(13)-\mathrm{O}(11)-\mathrm{Zn}$ & $129.6(3)$ & $\mathrm{O}(34)-\mathrm{Cl}-\mathrm{O}(32)$ & $122.9(10)$ \\
\hline $\mathrm{C}(23)-\mathrm{O}(21)-\mathrm{Zn}$ & $128.7(3)$ & $\mathrm{O}(34)-\mathrm{Cl}-\mathrm{O}(31)$ & $124.7(11)$ \\
\hline $\mathrm{N}(1)-\mathrm{C}(2)-\mathrm{C}(1)$ & $109.9(5)$ & $\mathrm{O}(32)-\mathrm{Cl}-\mathrm{O}(31)$ & 109.2(7) \\
\hline $\mathrm{C}(3)-\mathrm{N}(1)-\mathrm{C}(2)$ & $126.0(4)$ & $\mathrm{O}(34)-\mathrm{Cl}-\mathrm{O}(33)$ & $85.0(9)$ \\
\hline $\mathrm{O}(1)-\mathrm{C}(3)-\mathrm{N}(1)$ & $120.6(4)$ & $\mathrm{O}(32)-\mathrm{Cl}-\mathrm{O}(33)$ & $106.6(8)$ \\
\hline $\mathrm{O}(1)-\mathrm{C}(3)-\mathrm{N}(2)$ & $120.5(4)$ & $\mathrm{O}(31)-\mathrm{Cl}-\mathrm{O}(33)$ & $97.5(8)$ \\
\hline $\mathrm{N}(1)-\mathrm{C}(3)-\mathrm{N}(2)$ & $118.9(4)$ & & \\
\hline
\end{tabular}

Symmetry transformation used to generate equivalent atoms: $\# 1-x,-y,-z$.

TABle 6: Dimensions of the unique hydrogen bonds (distances in $\AA$ and angles in ${ }^{\circ}$ ) for complex $1 .{ }^{\dagger}$

\begin{tabular}{lccc}
\hline $\mathrm{D}^{\ddagger}-\mathrm{H} \cdots \mathrm{A}^{\S}$ & $\mathrm{D}^{\ddagger} \cdots \mathrm{A}^{\S}$ & $\mathrm{H} \cdots \mathrm{A}^{\S}$ & $<\mathrm{D}^{\ddagger} \mathrm{HA}^{\S}$ \\
\hline $\mathrm{N}(1)-\mathrm{H}(1) \cdots \mathrm{O}(11) \mathrm{a}$ & $2.952(1)$ & $2.177(1)$ & $158.68(3)$ \\
$\mathrm{N}(11)-\mathrm{H}(11) \cdots \mathrm{O}(21) \mathrm{a}$ & $2.878(1)$ & $1.983(1)$ & $156.88(2)$ \\
$\mathrm{N}(21)-\mathrm{H}(21) \cdots \mathrm{O}(1)$ & $2.861(1)$ & $2.060(1)$ & $160.05(3)$ \\
$\mathrm{N}(2)-\mathrm{H}(2) \cdots \mathrm{F}(1) \mathrm{b}$ & $2.930(1)$ & $2.272(1)$ & $159.06(3)$ \\
$\mathrm{N}(12)-\mathrm{H}(12) \cdots \mathrm{F}(2) \mathrm{c}$ & $2.947(1)$ & $2.135(1)$ & $163.27(3)$ \\
$\mathrm{N}(22)-\mathrm{H}(22) \cdots \mathrm{F}(3)$ & $2.964(1)$ & $151.44(2)$ \\
\hline
\end{tabular}

${ }^{\dagger}$ Symmetry transformation used to generate equivalent atoms: $\mathrm{a}-x,-y,-z ; \mathrm{b} 1-x, 0.5+y, 0.5-z ; \mathrm{c} 1-x,-y, 1-z$.

${ }^{\ddagger} \mathrm{D}=$ donor atom.

$\S_{\mathrm{A}}=$ acceptor atom. 
TABle 7: Dimensions of the unique hydrogen bonds (distances in $\AA$ and angles in ${ }^{\circ}$ ) for complex $2 .{ }^{\dagger}$

\begin{tabular}{lccc}
\hline $\mathrm{D}^{\ddagger}-\mathrm{H} \cdots \mathrm{A}^{\S}$ & $\mathrm{D}^{\ddagger} \cdots \mathrm{A}^{\S}$ & $\mathrm{H} \cdots \mathrm{A}^{\S}$ & $<\mathrm{D}^{\ddagger} \mathrm{HA}^{\S}$ \\
\hline $\mathrm{N}(2)-\mathrm{H}(2) \cdots \mathrm{O}(21)$ & $2.905(1)$ & $2.154(1)$ & $155.07(4)$ \\
$\mathrm{N}(12)-\mathrm{H}(12) \cdots \mathrm{O}(1) \mathrm{a}$ & $2.914(1)$ & $2.177(1)$ & $159.29(3)$ \\
$\mathrm{N}(22)-\mathrm{H}(22) \cdots \mathrm{O}(11) \mathrm{a}$ & $2.908(1)$ & $2.153(1)$ & $153.49(2)$ \\
$\mathrm{N}(1)-\mathrm{H}(1) \cdots \mathrm{O}(31) \mathrm{b}$ & $3.081(1)$ & $2.381(1)$ & $151.68(3)$ \\
$\mathrm{N}(11)-\mathrm{H}(11) \cdots \mathrm{O}(32)$ & $3.013(1)$ & $2.234(1)$ & $150.97(2)$ \\
$\mathrm{N}(21)-\mathrm{H}(21) \cdots \mathrm{O}(33) \mathrm{c}$ & $3.072(1)$ & $2.386(1)$ & $160.41(5)$ \\
\hline
\end{tabular}

${ }^{\dagger}$ Symmetry transformation used to generate equivalent atoms: $\mathrm{a}-x,-y,-z ; \mathrm{b}-x, 1-y, 1-z ; \mathrm{c} 1-x, 1-y, 1-z$.

${ }^{\ddagger} \mathrm{D}=$ donor atom.

$\S \mathrm{A}=$ acceptor atom.

TABle 8: Dimensions of the unique hydrogen bonds (distances in $\AA$ and angles in ${ }^{\circ}$ ) for complex $3 .{ }^{\dagger}$

\begin{tabular}{lccc}
\hline $\mathrm{D}^{\ddagger}-\mathrm{H} \cdots \mathrm{A}^{\S}$ & $\mathrm{D}^{\ddagger} \cdots \mathrm{A}^{\S}$ & $\mathrm{H} \cdots \mathrm{A}^{\S}$ & $<\mathrm{D}^{\ddagger} \mathrm{HA}^{\S}$ \\
\hline $\mathrm{N}(1)-\mathrm{H}(1) \cdots \mathrm{O}(11)$ & $2.886(1)$ & $2.055(1)$ & $156.94(5)$ \\
$\mathrm{N}(11)-\mathrm{H}(11) \cdots \mathrm{O}(21) \mathrm{a}$ & $2.892(1)$ & $2.082(1)$ & $155.01(5)$ \\
$\mathrm{N}(21)-\mathrm{H}(21) \cdots \mathrm{O}(1) \mathrm{a}$ & $2.890(2)$ & $2.091(1)$ & $154.16(6)$ \\
$\mathrm{N}(2)-\mathrm{H}(2) \cdots \mathrm{O}(31)$ & $3.097(1)$ & $2.343(1)$ & $159.73(6)$ \\
$\mathrm{N}(12)-\mathrm{H}(12) \cdots \mathrm{O}(33) \mathrm{b}$ & $3.077(2)$ & $2.369(1)$ & $156.88(5)$ \\
$\mathrm{N}(22)-\mathrm{H}(22) \cdots \mathrm{O}(32) \mathrm{c}$ & $3.038(1)$ & $144.26(5)$ \\
\hline
\end{tabular}

${ }^{\dagger}$ Symmetry transformation used to generate equivalent atoms: $\mathrm{a}-x,-y,-z ; \mathrm{b} x, y, 1+z ; \mathrm{c} 1-x, 1-y,-z$.

${ }^{\ddagger} \mathrm{D}=$ donor atom.

$\S_{\mathrm{A}}=$ acceptor atom.

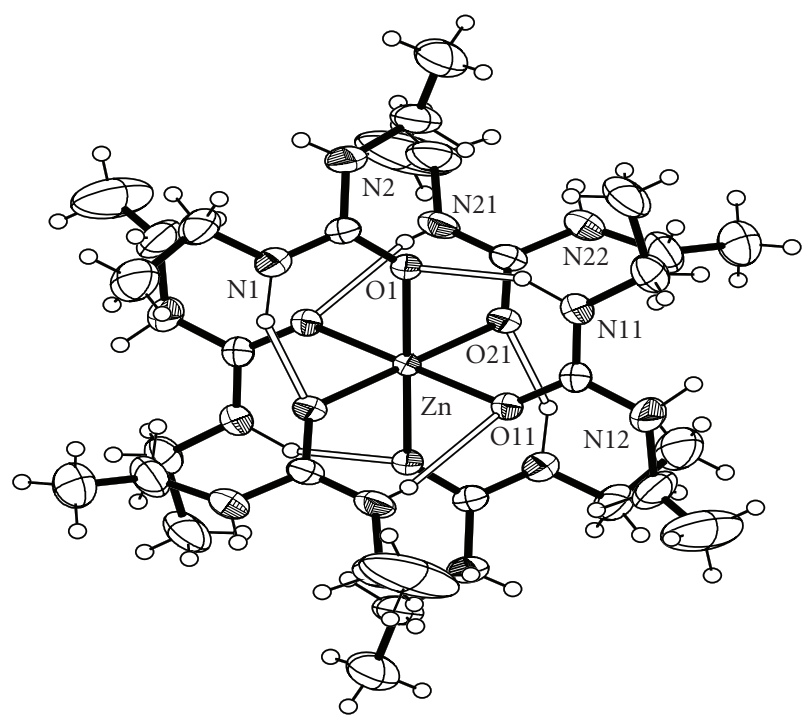

FIgURE 4: An ORTEP representation of the cation $\left[\mathrm{Co}(\mathrm{DEU})_{6}\right]^{2+}$ present in complex 4. Open bonds indicate intramolecular hydrogen bonds. The symmetry-equivalent atoms are not labeled.

network with a rutile (rtl) topology $[30,31]$ and Schläfli symbol $\left(4.6^{2}\right)_{2}\left(4^{2} .6^{10} .8^{3}\right)$ (Figure 8 ). It is worth noting that none of the $\left[\mathrm{M}(\mathrm{DMU})_{6}\right] \mathrm{X}_{2}$ complexes $[17,18]$ adopts a $3 \mathrm{D}$ net.

3.3. Vibrational Spectra of the Complexes. Table 10 gives diagnostic IR bands of the free ligand and complexes 14. Assignments have been given in comparison with the

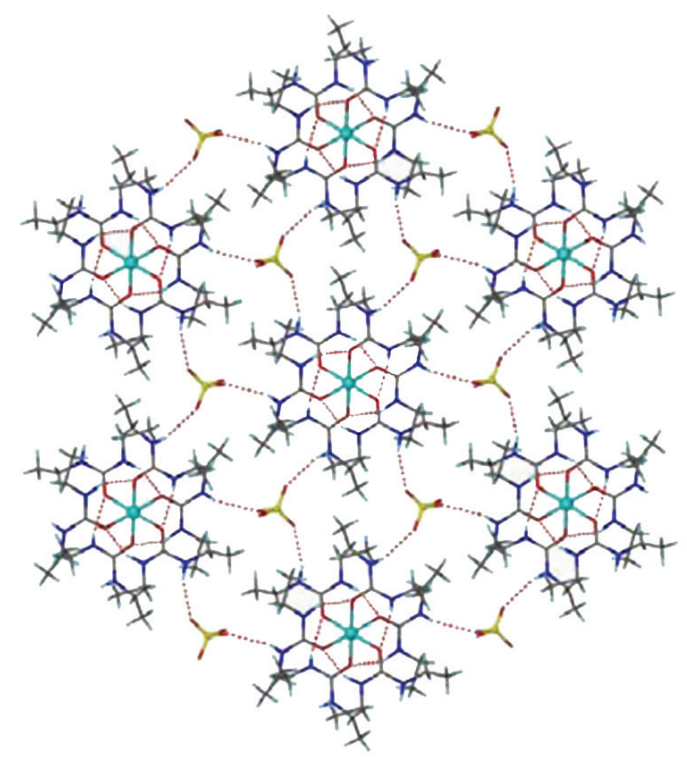

FIGURE 5: A view of the 2D framework formed by hydrogen bonding between the $\left[\mathrm{Zn}(\mathrm{DEU})_{6}\right]^{2+}$ cations and the $\mathrm{ClO}_{4}{ }^{-}$anions in 4 . The same framework is adopted by complexes 2 and 3 .

data obtained for the free DMU [32], the free DEU [33] and its $\mathrm{Co}(\mathrm{II})$ and $\mathrm{Ni}(\mathrm{II})$ complexes [34]. The bands with $v(\mathrm{CN})$ character are situated at higher wavenumbers in the spectra of 1-4 than for free DEU, whereas the $v(\mathrm{CO})$ band shows a frequency decrease. These shifts are consistent with oxygen coordination, suggesting the presence of ${ }^{+} \mathrm{N}=\mathrm{C}-\mathrm{O}^{-}$ 
TABle 9: Dimensions of the unique hydrogen bonds (distances in $\AA$ and angles in ${ }^{\circ}$ ) for complex $4 .^{\dagger}$

\begin{tabular}{lccc}
\hline $\mathrm{D}^{\ddagger}-\mathrm{H} \cdots \mathrm{A}^{\S}$ & $\mathrm{D}^{\ddagger} \cdots \mathrm{A}^{\S}$ & $\mathrm{H} \cdots \mathrm{A}^{\S}$ & $<\mathrm{D}^{\ddagger} \mathrm{HA}^{\S}$ \\
\hline $\mathrm{N}(1)-\mathrm{H}(1) \cdots \mathrm{O}(21) \mathrm{a}$ & $2.905(2)$ & $1.876(1)$ & $150.35(6)$ \\
$\mathrm{N}(11)-\mathrm{H}(11) \cdots \mathrm{O}(1)$ & $2.904(2)$ & $1.932(1)$ & $152.80(6)$ \\
$\mathrm{N}(21)-\mathrm{H}(21) \cdots \mathrm{O}(11) \mathrm{a}$ & $2.921(2)$ & $2.314(2)$ & $162.75(10)$ \\
$\mathrm{N}(2)-\mathrm{H}(2) \cdots \mathrm{O}(33)$ & $3.153(2)$ & $2.392(2)$ & $160.19(8)$ \\
$\mathrm{N}(12)-\mathrm{H}(12) \cdots \mathrm{O}(31) \mathrm{b}$ & $3.086(2)$ & $2.234(1)$ & $155.95(7)$ \\
$\mathrm{N}(22)-\mathrm{H}(22) \cdots \mathrm{O}(32) \mathrm{c}$ & $3.035(2)$ & $2.365(1)$ & $150.81(8)$ \\
\hline
\end{tabular}

${ }^{\dagger}$ Symmetry transformation used to generate equivalent atoms: a $-x,-y,-z ; \mathrm{b} x, y, 1-z ; \mathrm{c} 1-x, 1-y, 1-z$.

${ }^{\ddagger} \mathrm{D}=$ donor atom.

$\S_{\mathrm{A}}=$ acceptor atom.

TABLE 10: Most characteristic and diagnostic IR fundamentals $\left(\mathrm{cm}^{-1}\right)$ for DEU and complexes $\mathbf{1}-\mathbf{4} .^{a}$

\begin{tabular}{lccccc}
\hline Assignments & DEU & $\mathbf{1}$ & $\mathbf{2}$ & $\mathbf{3}$ & $\mathbf{4}$ \\
\hline$\nu(\mathrm{NH})$ & $3342 \mathrm{sb}$ & $3332 \mathrm{sb}$ & $3332 \mathrm{sb}$ & $3328 \mathrm{sb}$ & $3340 \mathrm{sb}$ \\
$\nu(\mathrm{CH})$ & $2973 \mathrm{~s}, 2932 \mathrm{~m}$, & $2976 \mathrm{~s}, 2934 \mathrm{~m}$, & $2972 \mathrm{~s}, 2934 \mathrm{~m}$, & $2976 \mathrm{~m}, 2934 \mathrm{w}$, & $2972 \mathrm{~s}, 2932 \mathrm{~m}$, \\
& $2874 \mathrm{~m}$ & $2878 \mathrm{~m}$ & $2876 \mathrm{w}$ & $2876 \mathrm{w}$ & $2876 \mathrm{w}$ \\
as $(\mathrm{CN})_{\text {amide }}+\delta_{\text {as }} \nu(\mathrm{NH})$ & $1625 \mathrm{vs}$ & $1576 \mathrm{vs}$ & $1570 \mathrm{vs}$ & $1570 \mathrm{vs}$ & $1682 \mathrm{vs}$ \\
$\nu(\mathrm{CO})$ & $1586 \mathrm{vs}$ & $1626 \mathrm{vs}$ & $1628 \mathrm{vs}$ & $1450 \mathrm{~m}$ & $1624 \mathrm{vs}$ \\
$\delta_{\mathrm{s}}(\mathrm{NH})$ & $1540 \mathrm{~m} \mathrm{sh}$ & $1454 \mathrm{~m}$ & $1452 \mathrm{w}$ & $1354 \mathrm{w}$ & $1334 \mathrm{w}$ \\
$\delta_{\text {as }}(\mathrm{NH})+{ }_{\text {as }}(\mathrm{CN})_{\text {amide }} \nu$ & $1259 \mathrm{~m}$ & $1338 \mathrm{~m}$ & $1338 \mathrm{w}$ & 1334 \\
\hline
\end{tabular}

${ }^{\mathrm{a}} \mathrm{KBr}$ pellets.

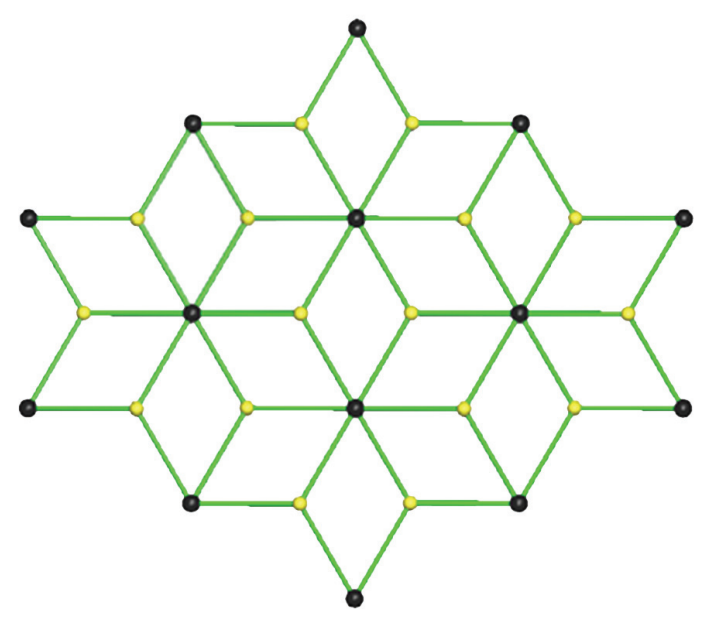

Figure 6: A view of the 2D hydrogen-bonded kgd- $\left(4^{3}\right)_{2}\left(4^{6} \cdot 6^{6} \cdot 8^{3}\right)$ net adopted by complexes $2-4$. Black spheres represent the 6connected $\left[\mathrm{M}(\mathrm{DEU})_{6}\right]^{2+}$ cations $[\mathrm{M}=\mathrm{Co}(2), \mathrm{Ni}(3)$ and $\mathrm{Zn}(4)]$ and yellow spheres the 3 -connected $\mathrm{ClO}_{4}{ }^{-}$anions.

resonant forms $[17,18]$. Upon coordination via oxygen, the positively charged metal ion stabilizes the negative charge on the oxygen atom; the NCO group now occurs in its polar resonance form and the double bond character of the $\mathrm{CN}$ bond increases, while the double bond character of the $\mathrm{CO}$ bond decreases, resulting in an increase of the $\mathrm{CN}$ stretching frequency with a simultaneous decrease in the CO stretching frequency $[17,18]$. The $v_{3}\left(\mathrm{~F}_{2}\right)\left[\nu_{d}(\mathrm{BF})\right]$ and $\nu_{4}\left(\mathrm{~F}_{2}\right)\left[\delta_{d}(\mathrm{FBF})\right]$ vibrations of the tetrahedral (point group $\left.\mathrm{T}_{d}\right) \mathrm{BF}_{4}^{-}$anion appear at $1100-1000$ and at 522$580 \mathrm{~cm}^{-1}$ (broad bands), respectively, in the IR spectrum of 1 [35]. The IR spectra of 2-4 exhibit strong bands at $\sim 1100$ and $626 \mathrm{~cm}^{-1}$ due to the $\nu_{3}\left(\mathrm{~F}_{2}\right)$ and $\nu_{4}\left(\mathrm{~F}_{2}\right)$ vibrations, respectively, of the uncoordinated $\mathrm{ClO}_{4}{ }^{-}$[35]. The broad character and splitting of the band at $\sim 1100 \mathrm{~cm}^{-1}$ indicate the involvement of the $\mathrm{ClO}_{4}{ }^{-}$ion in hydrogen bonding as it was established crystallographically (see above).

\section{Conclusions}

Following our studies on the coordination chemistry of urea (U) and N,N'-dimethylurea (DMU), N,N'-diethylurea (DEU) was employed as a ligand to form the stable octahedral complexes $\left[\mathrm{M}(\mathrm{DEU})_{6}\right]^{2+}$ with cobalt(II), nickel(II) and zinc(II). The structural characteristics of the $\left[\mathrm{M}(\mathrm{DEU})_{6}\right]^{2+}$ cation are very similar to the DMU analogs, that is, $\left[\mathrm{M}(\mathrm{DMU})_{6}\right]^{2+}$. All six DEU molecules are coordinated to metal centre in a bent fashion forming a $\mathrm{C}=\mathrm{O} \cdots \mathrm{M}$ angle of $\sim 130^{\circ}$, while six strong intracationic N-H $\cdots \mathrm{O}_{(\mathrm{DEU})}$ hydrogen bonds stabilize the $\left[\mathrm{M}(\mathrm{DEU})_{6}\right]^{2+}$ cations by creating six six-membered pseudochelate rings. The $\left[\mathrm{M}(\mathrm{DEU})_{6}\right]^{2+}$ cations and counterions $\left(\mathrm{ClO}_{4}{ }^{-}\right.$or $\left.\mathrm{BF}_{4}{ }^{-}\right)$self-assemble to form extended hydrogen-bonded architectures via 3 unique $\mathrm{N}-\mathrm{H} \cdots \mathrm{X}$ hydrogen bonds, $\left(\mathrm{X}=\mathrm{O}_{\text {(perchlorate) }}\right.$ or $\left.\mathrm{F}_{\text {(tetrafluoroborate) }}\right)$. The nature of the resulting supramolecular architectures is influenced by the nature of the counter-ion since the presence of $\mathrm{ClO}_{4}{ }^{-}$counter-ions gives rise to the formation of $2 \mathrm{D}$ hydrogen-bonded networks that conform to the kgd net while the presence of $\mathrm{BF}_{4}{ }^{-}$counter-ions results in a 3D hydrogen-bonded net with an rtl topology. By comparing the supramolecular architectures of the $\left[\mathrm{M}(\mathrm{DEU})_{6}\right] \mathrm{X}_{2}$ 
(a)

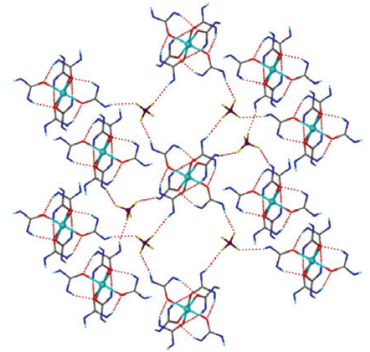

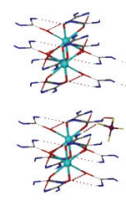

(b)
FIGURE 7: Views of the 3D framework formed by hydrogen bonds between the $\left[\mathrm{Co}(\mathrm{DEU})_{6}\right]^{2+}$ cations and the $\mathrm{BF}_{4}{ }^{-}$anions in $\mathbf{1}$.

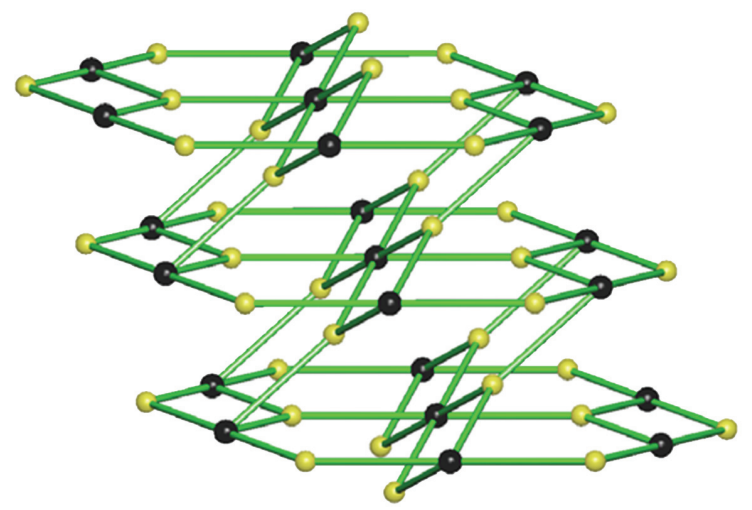

Figure 8: A view of the binodal 3D hydrogen-bonded rtl$\left(4.6^{2}\right)_{2}\left(4^{2} .6^{10} .8^{3}\right)$ net that 1 adopts. Black spheres represent the 6-connected $\left[\mathrm{Co}(\mathrm{DEU})_{6}\right]^{2+}$ cations and yellow spheres the 3connected $\mathrm{BF}_{4}{ }^{-}$anions.

$\left(\mathrm{X}=\mathrm{ClO}_{4}\right.$ or $\left.\mathrm{BF}_{4}\right)$ and the $\left[\mathrm{M}(\mathrm{DMU})_{6}\right] \mathrm{X}_{2}\left(\mathrm{X}=\mathrm{ClO}_{4}\right.$ or $\left.\mathrm{BF}_{4}\right)$ we can conclude that the substitution of DMU by DEU considerably affected the nature of the hydrogen-bonded networks. We are presently pursuing our studies on the coordination chemistry of urea and its symmetrically or unsymmetrically substituted alkyl derivatives to generate a rich variety of hydrogen-bonded networks.

\section{Acknowledgment}

G. S. Papaefstathiou thanks the Special Account for Research Grants (SARG) of the National and Kapodistrian University of Athens for funding this paper.

\section{References}

[1] K. C. Nicolaou and T. Montagnon, Molecules that Changed the World, Wiley-VCH, Weinheim, Germany, 2008.

[2] W. F. Boron and E. L. Boulpaep, Medical Physiology, Updated Edition, Saunders, Philadelphia, Pa, USA, 2004.

[3] J. H. Meessen and H. Petersen, "Urea," in Ullmann's Encyclopedia of Industrial Chemistry, Electronic Release, Wiley-VCH, Weinheim, Germany, 6th edition, 2002.

[4] F. Meyer, M. Konrad, and E. Kaifer, "Novel $\mu_{3}$-coordination of urea at a nickel(II) site: structure, reactivity and ferromagnetic superexchange," European Journal of Inorganic Chemistry, no. 11, pp. 1851-1854, 1999.

[5] H. E. Wages, K. L. Taft, and S. J. Lippard, " $\left.\left[\mathrm{Ni}_{2}(\mathrm{OAc})_{3} \text { (urea)(tmen }\right)_{2}\right](\mathrm{OTf})$ and $\left[\mathrm{Ni}(\mathrm{OAc})(\text { urea })_{2}\right.$ (tmen)](OTf), model complexes for the enzyme urease," Inorganic Chemistry, vol. 32, no. 23, pp. 4985-4987, 1993.

[6] T. Theophanides and P. D. Harvey, "Structural and spectroscopic properties of metal-urea complexes," Coordination Chemistry Reviews, vol. 76, pp. 237-264, 1987.

[7] P. S. Gentile, P. Carfagno, S. Haddad, and L. Campisi, "The preparation of the bidentate urea complex $\left[\mathrm{Co}(\text { urea })_{4}\right]\left(\mathrm{NO}_{3}\right)_{2}$," Inorganica Chimica Acta, vol. 6, no. C, pp. 296-298, 1972.

[8] D. S. Sagatys, R. C. Bott, G. Smith, K. A. Byriel, and C. H. L. Kennard, "The preparation and crystal structure of a polymeric $(1: 1)$-silver nitrate-urea complex, $\left[\left(\mathrm{AgNO}_{3}\right)_{2}\left(\mathrm{CH}_{4} \mathrm{~N}_{2} \mathrm{O}\right)_{2}\right]_{n}$," Polyhedron, vol. 11, no. 1, pp. 49$52,1992$.

[9] K. Lewinski, J. Sliwinski, and L. Lebioda, "Structure of (urea)mercury(II) chloride and the effects of strain on ligand properties of urea," Inorganic Chemistry, vol. 22, no. 16, pp. 2339-2342, 1983.

[10] S. V. Kryatov, A. Y. Nazarenko, P. D. Robinson, and E. V. Rybak-Akimova, "A dinuclear iron (III) complex with a bridging urea anion: implications for the urease mechanism," Chemical Communications, no. 11, pp. 921-922, 2000.

[11] S. Subramanian and M. J. Zaworotko, "Exploitation of the hydrogen bond: recent developments in the context of crystal engineering," Coordination Chemistry Reviews, vol. 137, pp. 357-401, 1994.

[12] X. Zhao, Y.-L. Chang, F. W. Fowler, and J. W. Lauher, "An approach to the design of molecular solids. The ureylenedicarboxylic acids," Journal of the American Chemical Society, vol. 112, no. 18, pp. 6627-6634, 1990.

[13] T. L. Nguyen, F. W. Fowler, and J. W. Lauher, "Commensurate and incommensurate hydrogen bonds. An exercise in crystal engineering," Journal of the American Chemical Society, vol. 123, no. 44, pp. 11057-11064, 2001.

[14] P. S. Corbin, S. C. Zimmerman, P. A. Thiessen, N. A. Hawryluk, and T. J. Murray, "Complexation-induced unfolding of heterocyclic ureas. Simple foldamers equilibrate with multiply hydrogen-bonded sheetlike structures," Journal of the American Chemical Society, vol. 123, no. 43, pp. 10475-10488, 2001.

[15] S. Swaminathan and B. M. Craven, "The crystal structure and molecular thermal motion of urea at 12,60 and $123 \mathrm{~K}$ from neutron diffraction," Acta Crystallographica, vol. B40, pp. 300306,1984

[16] E. Manessi-Zoupa and G. S. Papaefstathiou, "Synthesis, xray structure and characterization of a complex containing the hexakis(urea)cobalt(II) cation and lattice urea molecules," Bioinorganic Chemistry and Applications, vol. 2007, Article ID 51567, 7 pages, 2007.

[17] R. Keuleers, H. O. Desseyn, G. S. Papaefstathiou et al., "Hydrogen-bonded networks based on manganese(II), nickel(II), copper(II) and zinc(II) complexes of N,N'dimethylurea," Transition Metal Chemistry, vol. 28, no. 5, pp. 548-557, 2003.

[18] G. S. Papaefstathiou, R. Keuleers, C. J. Milios et al., "The hexakis(N,N'-dimethylurea)cobalt(II) cation: a flexible building block for the construction of hydrogen bonded networks," Zeitschrift für Naturforschung, vol. 58b, no. 1, pp. 74-84, 2003. 
[19] E. Diamantopoulou, G. S. Papaefstathiou, A. Terzis, C. P. Raptopoulou, H. O. Desseyn, and S. P. Perlepes, "Hydrogen bonded networks based on lanthanide(III) complexes of $\mathrm{N}, \mathrm{N}^{\prime}$-dimethylurea (DMU): preparation, characterisation, and crystal structures of $\left[\mathrm{Nd}(\mathrm{DMU})_{6}\right]\left[\mathrm{NdCl}_{6}\right]$ and $\left[\mathrm{Nd}\left(\mathrm{NO}_{3}\right)_{3}(\mathrm{DMU})_{3}\right]$," Polyhedron, vol. 22, no. 6, pp. 825-835, 2003.

[20] R. Keuleers, G. S. Papaefstathiou, C. P. Raptopoulou, S. P. Perlepes, and H. O. Desseyn, "Comparative study of the metalligand bond strength in $\mathrm{Mn}^{\mathrm{II}} / \mathrm{X} / \mathrm{U}$ complexes $(\mathrm{X}=\mathrm{Cl}, \mathrm{Br}, \mathrm{I}$; $\mathrm{U}$ = urea)," Journal of Molecular Structure, vol. 525, no. 1-3, pp. 173-183, 2000.

[21] R. Keuleers, G. S. Papaefstathiou, C. P. Raptopoulou, V. Tangoulis, H. O. Desseyn, and S. P. Perlepes, “Tris $\left(N, N^{\prime}\right.$ dimethylurea)bis(nitrato- $O, \mathrm{O}^{\prime}$ ) manganese(II), the first example of a seven-coordinate manganese (II) complex with a monodentate organic ligand," Inorganic Chemistry Communications, vol. 2, no. 10, pp. 472-475, 1999.

[22] S. Calogero, U. Russo, G. Valle, P. W. C. Barnard, and J. D. Donaldson, "A study of cis-octahedral compounds of tin(IV) halides with thiourea or urea type ligands," Inorganica Chimica Acta, vol. 59, pp. 111-116, 1982.

[23] U. Russo, G. Valle, and S. Calogero, "Hexakis(diethylurea)iron(II) perchlorate, $\mathrm{C}_{30} \mathrm{H}_{72} \mathrm{Cl}_{2} \mathrm{FeN}_{12} \mathrm{O}_{14}$, Crystal Structure Communications, vol. 9, pp. 443-448, 1980.

[24] J. Delaunay and R. P. Hugel, "Molecular structure of hexakis(N,N'-diethylurea)manganese(II) tetrabromomanganate(II)," Inorganic Chemistry, vol. 28, no. 12, pp. 2482-2485, 1989.

[25] G. M. Sheldrick, SHELXS-86, Structure Solving Program, University of Göttingen, Göttingen, Germany, 1986.

[26] G. M. Sheldrick, SHELXL-97, Program for the Refinement of Crystal Structures from Diffraction Data, University of Göttingen, Göttingen, Germany, 1997.

[27] http://www.topos.ssu.samara.ru.

[28] V. A. Blatov, "Multipurpose crystallochemical analysis with the program package TOPOS," IUCr CompComm Newsletter, pp. $4-38,2006$.

[29] H. Irving and R. J. P. Williams, "The stability of transitionmetal complexes," Journal of the Chemical Society, pp. 31923210, 1953.

[30] M. O'Keeffe, M. Eddaoudi, H. Li, T. Reineke, and O. M. Yaghi, "Frameworks for extended solids: geometrical design principles," Journal of Solid State Chemistry, vol. 152, no. 1, pp. 3-20, 2000.

[31] O. D. Friedrichs, M. O’Keeffe, and O. M. Yaghi, “The $\mathrm{CdSO}_{4}$, rutile, cooperite and quartz dual nets: interpenetration and catenation," Solid State Sciences, vol. 5, no. 1, pp. 73-78, 2003.

[32] R. Keuleers, H. O. Desseyn, B. Rousseau, and C. Van Alsenoy, "Solids modeled by ab initio crystal field methods. 21. Study of the structure and vibrational spectrum of N,N'-dimethylurea in the gas phase and in its Cc crystal phase," Journal of Physical Chemistry A, vol. 104, no. 25, pp. 5946-5954, 2000.

[33] Y. Mido, "Infrared spectra and configurations of dialkylureas," Spectrochimica Acta Part A, vol. 28, no. 8, pp. 1503-1518, 1972.

[34] J. P. Barbier and R. Hugel, "New complexes of cobalt(II) and nickel(II) salts with N,N'-diethylurea," Inorganica Chimica Acta, vol. 18, no. C, pp. 253-256, 1976.

[35] K. Nakamoto, Infrared and Raman Spectra of Inorganic and Coordination Compounds, John Wiley and Sons, New York, NY, USA, 4th edition, 1986. 


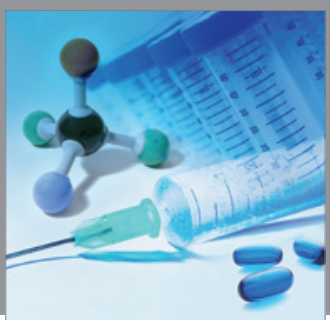

International Journal of

Medicinal Chemistry

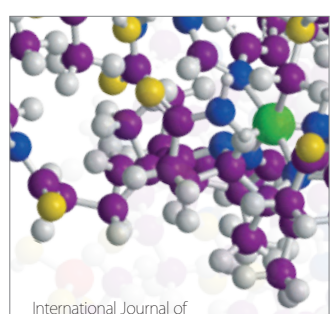

Carbohydrate Chemistry

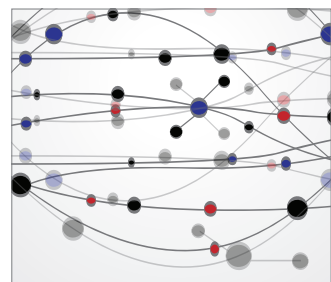

The Scientific World Journal
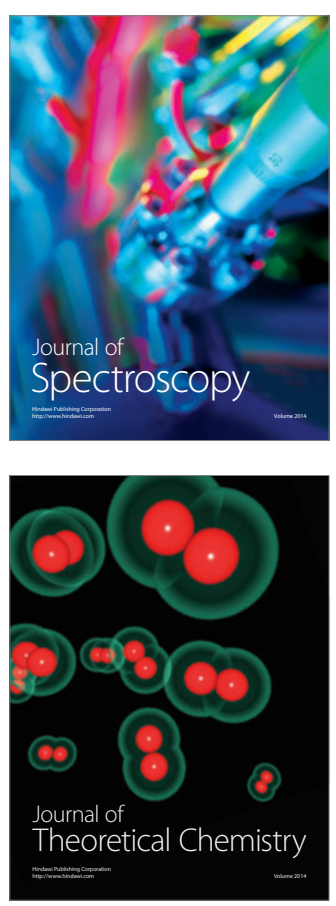
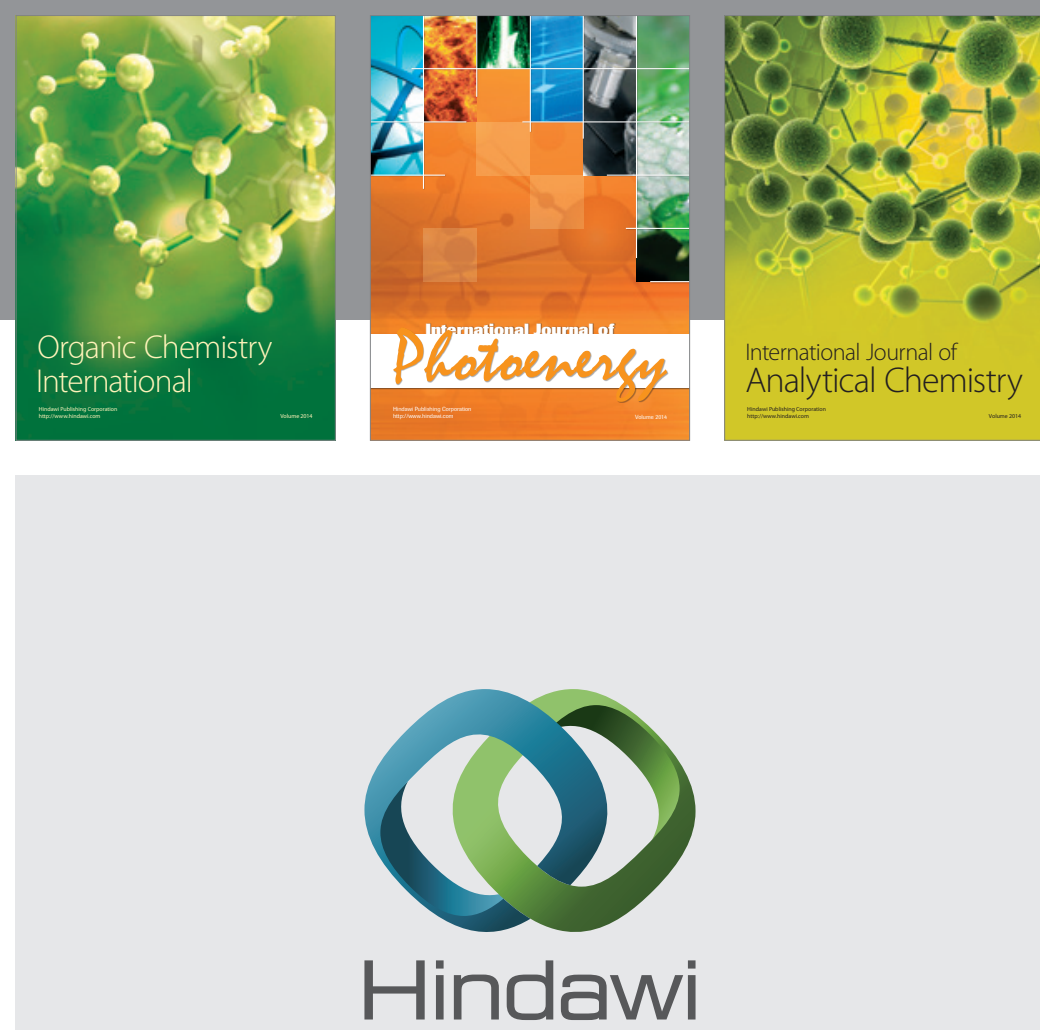

Submit your manuscripts at

http://www.hindawi.com
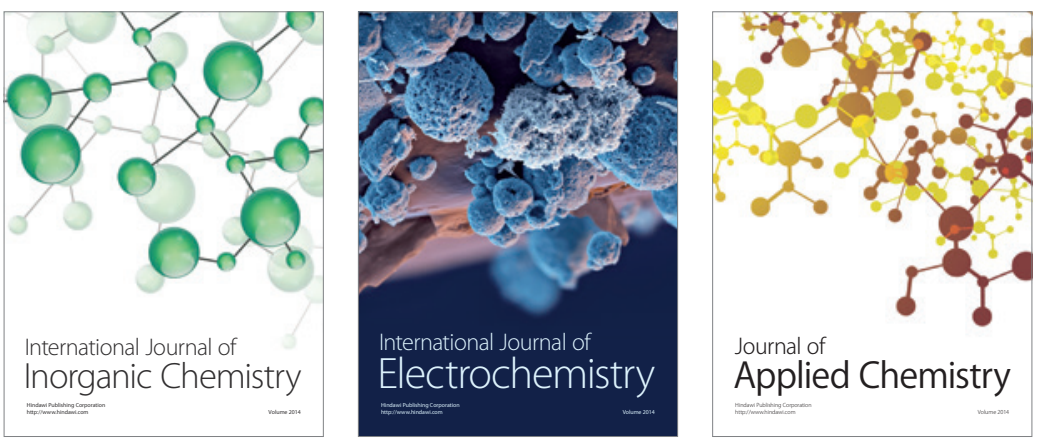

Journal of

Applied Chemistry
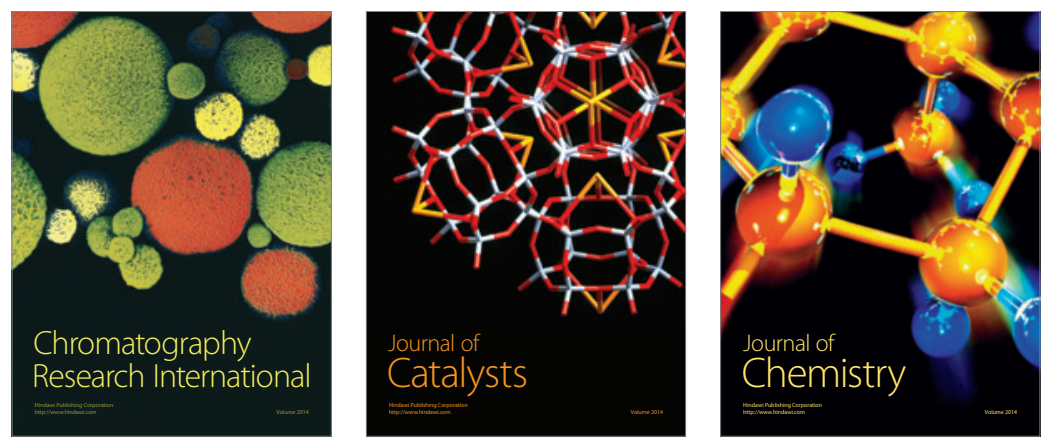
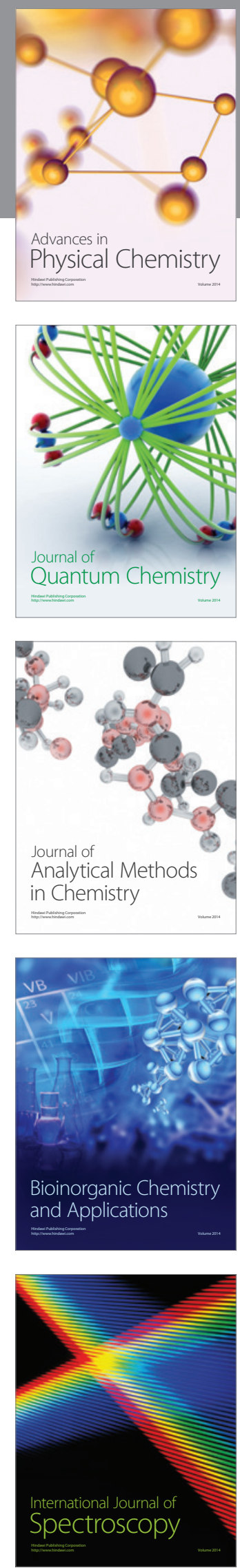